\title{
Some Generalized Forms of Fuzzy Interval Valued Hyperideals in a Hyperring
}

\author{
Hongjie $\mathrm{Li}^{1}{ }^{1}$ Zeyuan $\mathrm{Li}^{2}{ }^{2}$ and Yunqiang $\mathrm{Yin}^{3,4}$ \\ ${ }^{1}$ Mathematics Department, Zhoukou Normal University, Zhoukou 466001, China \\ ${ }^{2}$ College of Mechanical and Electrical Engineering, Harbin Institute of Technology, Harbin 150001, China \\ ${ }^{3}$ College of Sciences, East China Institute of Technology, Nanchang 330013, China \\ ${ }^{4}$ Faculty of Science, Kunming University of Science and Technology, Kunming 650093, China
}

Correspondence should be addressed to Hongjie Li; lhjjs10715201@163.com

Received 15 October 2013; Revised 20 May 2014; Accepted 22 May 2014; Published 12 June 2014

Academic Editor: Hector Pomares

Copyright (C) 2014 Hongjie Li et al. This is an open access article distributed under the Creative Commons Attribution License, which permits unrestricted use, distribution, and reproduction in any medium, provided the original work is properly cited.

\begin{abstract}
Some generalized forms of the hyperideals of a hyperring in the paper of Zhan et al. (2008) will be given. As a generalization of the interval valued $(\alpha, \beta)$-fuzzy hyperideals of a hyperring with $\alpha, \beta \in\{\epsilon, q, \in \wedge q, \in \vee q\}$ and $\alpha \neq \in \wedge q$, the notion of generalized interval valued $(\alpha, \beta)$-fuzzy hyperideals of a hyperring is also introduced. Special attention is concentrated on the interval valued $\left(\epsilon_{\tilde{\gamma}}, \epsilon_{\tilde{\gamma}} \vee q_{\tilde{\delta}}\right)$-fuzzy hyperideals. As a consequence, some characterizations theorems of interval valued $\left(\epsilon_{\tilde{\gamma}}, \epsilon_{\tilde{\gamma}} \vee q_{\tilde{\delta}}\right)$-fuzzy hyperideals will be provided.
\end{abstract}

\section{Introduction}

The concept of hyperstructure was first introduced by Marty [1] in 1934. People later observed that hyperstructures have many applications in pure and applied sciences. A comprehensive review of hyperstructures can be found in $[2,3]$. In a recent monograph of Corsini and Leoreanu [4], the authors have collected numerous applications of algebraic hyperstructures, especially those from the last decade to the following subjects: geometry, hypergraphs, binary relations, lattices, fuzzy sets and rough sets, automata, cryptography, codes, median algebras, relation algebras, artificial intelligence, and probabilities.

After introducing the concept of fuzzy sets of Zadeh in 1965 (see [5]), there are many papers devoted to fuzzify the classical mathematics into fuzzy mathematics. The relationships between the fuzzy sets and algebraic hyperstructures (structures) have been considered by Corsini, Davvaz, Kehagias, Leoreanu, Yin, Zahedi, Zhan, and others. The reader is referred to the papers [6-24]. By using the notion "belongingness $(\epsilon)$ " and "quasicoincidence $(q)$ " of a fuzzy point with a fuzzy set introduced by $\mathrm{Pu}$ and Liu [25], the concept of $(\alpha, \beta)$-fuzzy subgroups, where $\alpha, \beta$ are any two in $\{\epsilon, q, \in \vee q, \epsilon \wedge q\}$ with $\alpha \neq \in \wedge q$, was introduced by Bhakat and Das [26] in 1992, in which they first generalized Rosenfeld's fuzzy subgroup [27]. The detailed study of $(\epsilon$ $, \epsilon \vee q)$-fuzzy subgroups has been considered by Bhakat and Das in [28] and Bhakat in $[29,30]$. The concept of the $(\epsilon, \epsilon \vee q)$-fuzzy subhyperquasigroups of hyperquasigroups was introduced by Davvaz and Corsini [12]. By using the idea of "quasicoincidence" of a fuzzy interval value with an interval valued fuzzy set, Zhan et al. [21] introduced and investigated the notion of interval valued $(\alpha, \beta)$-fuzzy hyperideals of a hyperring.

As a generalization of the concepts of "belongingness $(\epsilon)$ " and "quasicoincidence $(q)$ " of a fuzzy point with a fuzzy set introduced by $\mathrm{Pu}$ and Liu [25], Yin and Zhan [31] introduced the concept of " $\gamma$-belongingness $\left(\epsilon_{\gamma}\right)$ " and " $\delta$ quasicoincidence $\left(q_{\delta}\right)$ " of a fuzzy point with a fuzzy set. Using these new concepts, Yin and Zhan [31] introduced the concepts of $(\alpha, \beta)$-fuzzy (implicative, positive implicative, and fantastic) filters and $(\bar{\beta}, \bar{\alpha})$-fuzzy (implicative, positive implicative, and fantastic) filters of BL-algebras, where $\alpha, \beta \in$ $\left\{\epsilon_{\gamma}, q_{\delta}, \epsilon_{\gamma} \wedge q_{\delta}, \epsilon_{\tilde{\gamma}} \vee q_{\tilde{\delta}}\right\}, \bar{\alpha}, \bar{\beta} \in\left\{\overline{\epsilon_{\gamma}}, \overline{q_{\delta}}, \overline{\epsilon_{\gamma}} \wedge \overline{q_{\delta}}, \overline{\epsilon_{\gamma}} \vee \overline{q_{\delta}}\right\}$ $\alpha \neq \epsilon_{\gamma} \wedge q_{\delta}$, and $\bar{\beta} \neq \overline{\epsilon_{\gamma}} \wedge \overline{q_{\delta}}$, and some related properties 
were investigated. This idea is continued and studied by $\mathrm{Ma}$ et al. [32], Ma and Zhan [33], Yin and Zhan [34], and so on. Following this idea, in this paper, we will give some of the generalized forms of the interval fuzzy hyperideals given by Zhan et al. in [21]. The notion of generalized interval valued $(\alpha, \beta)$-fuzzy hyperideals is introduced which is the generalization of the notion of interval valued $(\alpha, \beta)$-fuzzy hyperideals. Special attention will be paid to the interval valued $\left(\epsilon_{\tilde{\gamma}}, \epsilon_{\tilde{\gamma}} \vee q_{\tilde{\delta}}\right)$-fuzzy hyperideals. It is noteworthy that the notion of an interval valued $(\alpha, \beta)$-fuzzy hyperideal is a special generalized interval valued $(\alpha, \beta)$-fuzzy hyperideal and hence many results in [21] will become easy corollaries of our results given in this paper.

\section{Hyperrings}

We first recall that a hyperstructure is a nonempty set $H$ together with a mapping "o": $H \times H \rightarrow \mathbb{P}^{*}(H)$, where $\mathbb{P}^{*}(H)$ is the set of all nonempty subsets of $H$, written as $(x, y) \mapsto x \circ y$. We note that if $x \in H$ and $A, B$ are nonempty subsets of $H$, then by $A \circ B A \circ x$ and $x \circ B$, we mean that $A \circ B=\bigcup_{a \in A, b \in B} a \circ b, A \circ x=A \circ\{x\}$ and $x \circ B=\{x\} \circ B$, respectively.

Now, we call a hyperstructure $(H, \circ)$ a canonical hypergroup [35] if the following axioms are satisfied:

(1) for every $x, y, z \in H,(x \circ y) \circ z=x \circ(y \circ z)$;

(2) for every $x, y \in H, x \circ y=y \circ x$;

(3) there is a unique $0 \in H$ such that $0 \circ x=x$, for all $x \in H$;

(4) for every $x \in H$, there exists a unique element $x^{\prime} \in H$ such that $0 \in x \circ x^{\prime}$ (we call the element $x^{\prime}$ the opposite of $x)$.

Definition 1 (see [36]). A hyperring is an algebraic structure $(R,+, \cdot)$ which satisfies the following axioms.

(1) $(R,+)$ is a canonical hypergroup (we will write $-x$ for $\left.x^{\prime}\right)$.

(2) $(R, \cdot)$ is a semigroup having zero as a bilaterally absorbing element.

(3) The multiplication is distributive with respect to the hyperoperation "+."

Let $(R,+, \cdot)$ be a hyperring and let $A$ be a nonempty subset of $R$. Then $A$ is called a subhyperring of $R$ if $(A,+, \cdot)$ itself is a hyperring. In what follows, let $R$ denote a hyperring unless otherwise stated. A subhyperring $A$ of $R$ is a left (right) hyperideal of $R$ if $r a \in A(a r \in A)$, for all $r \in R$ and $a \in A$. $A$ subhyperring $A$ is called a hyperideal if $A$ is both left and right hyperideal.

\section{Interval Valued Fuzzy Sets}

By an interval number $\tilde{a}$ [37], we mean an interval $\left[a^{-}, a^{+}\right]$, where $0 \leq a^{-} \leq a^{+} \leq 1$. The set of all interval numbers is denoted by $D[0,1]$. The interval $[a, a]$ can be simply identified by the number $a$. For the interval numbers $\widetilde{a}_{i}=\left[a_{i}^{-}, a_{i}^{+}\right], \widetilde{b}_{i}=$ $\left[b_{i}^{-}, b_{i}^{+}\right] \in D[0,1], i \in I$, where $I$ is an index set, we define

$$
\begin{gathered}
\operatorname{rmax}\left\{\tilde{a}_{i}, \tilde{b}_{i}\right\}=\left[\max \left(a_{i}^{-}, b_{i}^{-}\right), \max \left(a_{i}^{+}, b_{i}^{+}\right)\right], \\
\operatorname{rmin}\left\{\tilde{a}_{i}, \tilde{b}_{i}\right\}=\left[\min \left(a_{i}^{-}, b_{i}^{-}\right), \min \left(a_{i}^{+}, b_{i}^{+}\right)\right], \\
\bigvee_{i \in I} \tilde{a}_{i}=\left[\bigvee_{i \in I} a_{i}^{-}, \bigvee_{i \in I} a_{i}^{+}\right], \\
\bigwedge_{i \in I} \tilde{a}_{i}=\left[\bigwedge_{i \in I} a_{i}^{-}, \bigwedge_{i \in I} a_{i}^{+}\right] .
\end{gathered}
$$

For any intervals $\tilde{a}=\left[a^{-}, a^{+}\right]$and $\tilde{b}=\left[b^{-}, b^{+}\right]$, where $0 \leq a^{-} \leq a^{+}$and $0 \leq b^{-} \leq b^{+}$, we define

$$
\begin{gathered}
\tilde{a}+\tilde{b}=\left[a^{-}+b^{-}, a^{+}+b^{+}\right], \\
\tilde{a}-\tilde{b}=\left[a^{-}-b^{-}, a^{+}-b^{+}\right], \quad \text { whenever } a^{-} \leq b^{-}, a^{+} \leq b^{+}, \\
k \tilde{a}=\left[k a^{-}, k a^{+}\right], \quad \text { whenever } k \geq 0,
\end{gathered}
$$

and we put

(i) $\tilde{a} \leq \widetilde{b} \Leftrightarrow a^{-} \leq b^{-}$and $a^{+} \leq b^{+}$,

(ii) $\tilde{a}=\tilde{b} \Leftrightarrow a^{-}=b^{-}$and $a^{+}=b^{+}$,

(iii) $\tilde{a}<\tilde{b} \Leftrightarrow \tilde{a} \leq \tilde{b}$ and $\tilde{a} \neq \tilde{b}$.

Denote by $D^{\prime}[0,1]$ the set all of interval numbers such that any two elements of $D^{\prime}[0,1]$ are comparable. Then, it is clear that both $(D[0,1], \leq, \wedge, \vee)$ and $\left(D[0,1]^{\prime}, \leq, \wedge, \vee\right)$ form a complete lattice with $0=[0,0]$ as their least element and $1=[1,1]$ as their greatest element.

Recall that an interval valued fuzzy subset $F$ on $X$ is the set

$$
F=\left\{\left(x,\left[\mu_{F}^{-}(x), \mu_{F}^{+}(x)\right]\right) \mid x \in X\right\},
$$

where $\mu_{F}^{-}$and $\mu_{F}^{+}$are two fuzzy subsets of $X$ such that $\mu_{F}^{-}(x) \leq$ $\mu_{F}^{+}(x)$ for all $x \in X$. Putting $\widetilde{\mu_{F}}(x)=\left[\mu_{F}^{-}(x), \mu_{F}^{+}(x)\right]$, we see that $F=\left\{\left(x, \widetilde{\mu_{F}}(x)\right) \mid x \in X\right\}$, where $\widetilde{\mu_{F}}: X \rightarrow D[0,1]$. Denote by $\mathbb{R}(X)$ the set of all interval valued fuzzy subsets of $X$ such that, for any $F \in \mathbb{R}(X), \operatorname{Im} F=\left\{\left[\mu_{F}^{-}(x), \mu_{F}^{+}(x)\right] \mid x \in\right.$ $X\} \subseteq D^{\prime}[0,1]$.

If $F$ and $G$ are two interval valued fuzzy subsets of $X$, then we define

$F \subseteq G$ if and only if, for all $x \in X, \mu_{F}^{-}(x) \leq \mu_{G}^{-}(x)$ and $\mu_{F}^{+}(x) \leq \mu_{G}^{+}(x)$,

$F=G$ if and only if, for all $x \in X, \mu_{F}^{-}(x)=\mu_{G}^{-}(x)$ and $\mu_{F}^{+}(x)=\mu_{G}^{+}(x)$.

Also, the union and intersection are defined as follows:

$$
\begin{gathered}
F \cup G=\left\{\left(x,\left[\max \left\{\mu_{F}^{-}(x), \mu_{G}^{-}(x)\right\},\right.\right.\right. \\
\left.\left.\left.\max \left\{\mu_{F}^{+}(x), \mu_{G}^{+}(x)\right\}\right]\right) \mid x \in X\right\}, \\
F \cap G=\left\{\left(x,\left[\min \left\{\mu_{F}^{-}(x), \mu_{G}^{-}(x)\right\},\right.\right.\right. \\
\left.\left.\left.\min \left\{\mu_{F}^{+}(x), \mu_{G}^{+}(x)\right\}\right]\right) \mid x \in X\right\} .
\end{gathered}
$$


An interval valued fuzzy subset $F$ of $X$ of the form

$$
\widetilde{\mu_{F}}(y)= \begin{cases}\widetilde{r}(\neq[0,0]) & \text { if } y=x, \\ {[0,0]} & \text { otherwise, }\end{cases}
$$

is said to be a fuzzy interval value with support $x$ and interval value $\widetilde{r}$ and is denoted by $U(x ; \widetilde{r})$.

Let $\tilde{\gamma}, \widetilde{\delta} \in D[0,1]$ be such that $\tilde{\gamma}<\widetilde{\delta}$. For a fuzzy interval value $U(x ; \widetilde{t})$ and an interval valued fuzzy set $F$, we say that

(1) $U(x ; \widetilde{r}) \epsilon_{\tilde{\gamma}} F$ if $\widetilde{\mu_{F}}(x) \geq \widetilde{r}>\widetilde{\gamma}$;

(2) $U(x ; \widetilde{r}) q_{\tilde{\delta}} F$ if $\widetilde{\mu_{F}}(x)+\widetilde{r}>2 \widetilde{\delta}$;

(3) $U(x ; \widetilde{r}) \epsilon_{\tilde{\gamma}} \vee q_{\tilde{\delta}} F$ if $U(x ; \widetilde{r}) \epsilon_{\tilde{\gamma}} F$ or $U(x ; \widetilde{r}) q_{\widetilde{\delta}} F$;

(4) $U(x ; \widetilde{r}) \epsilon_{\tilde{\gamma}} \wedge q_{\widetilde{\delta}} F$ if $U(x ; \widetilde{r}) \epsilon_{\tilde{\gamma}} F$ and $U(x ; \widetilde{r}) q_{\widetilde{\delta}} F$;

(5) $U(x ; \tilde{r}) \bar{\alpha} F$ if $U(x ; \widetilde{r}) \alpha F$ does not hold for $\alpha \epsilon$ $\left\{\epsilon_{\tilde{\gamma}}, q_{\tilde{\delta}}, \epsilon_{\tilde{\gamma}} \vee q_{\tilde{\delta}}, \epsilon_{\tilde{\gamma}} \wedge q_{\tilde{\delta}}\right\}$

Next we define a new ordering relation " $\subseteq \vee q_{(\tilde{\gamma}, \widetilde{\delta})}$ " on $\square \mathbb{F}(X)$, which is called the interval valued fuzzy inclusion or quasicoincidence relation, as follows.

For any $F, G \in \mathbb{E}(X)$ and $\tilde{\gamma}, \widetilde{\delta} \in[0,1]$ such that $\tilde{\gamma}<\widetilde{\delta}$, by $F \subseteq \vee q_{(\tilde{\gamma}, \tilde{\delta})} G$ we mean that $U(x ; \widetilde{r}) \epsilon_{\tilde{\gamma}} F \Rightarrow U(x ; \widetilde{r}) \epsilon_{\tilde{\gamma}} \vee$ $q_{\tilde{\delta}} G$ for all $x \in X$ and $\tilde{\gamma}<\tilde{r} \leq[1,1]$.

And we define a relation " $\approx$ " on $\mathbb{\square}(X)$ as follows.

For any $F, G \in \mathbb{R}(X)$, by $F \approx G$ we mean that $F \subseteq$ $\vee q_{(\tilde{\gamma}, \tilde{\delta})} G$ and $G \subseteq \vee q_{(\tilde{\gamma}, \tilde{\delta})} F$.

In the sequel, unless otherwise stated, we let $R$ be a hyperring, $\tilde{\gamma}, \widetilde{\delta} \in D[0,1]$, such that $\tilde{\gamma}<\widetilde{\delta}$ and denote by $\subseteq \vee q_{(\tilde{\gamma}, \tilde{\delta})}$ that $\subseteq \vee q_{(\tilde{\gamma}, \tilde{\delta})}$ is not true. We also emphasize that any interval valued fuzzy subset $F$ of $X$ must satisfy the following conditions:

$\widetilde{\mu_{F}}(x) \leq \tilde{\gamma}$ or $\tilde{\gamma}<\widetilde{\mu_{F}}(x)$ and $\widetilde{\mu_{F}}(x) \leq \tilde{\delta}$ or $\tilde{\delta}<\widetilde{\mu_{F}}(x)$ for all $x \in X$.

Lemma 2. Let $X$ be a nonempty set and $F, G \in \mathbb{F}(X)$. Then $F \subseteq \vee q_{(\widetilde{\gamma}, \tilde{\delta})} G$ if and only if $\operatorname{rmax}\left\{\widetilde{\mu_{G}}(x), \widetilde{\gamma}\right\} \geq$ $\operatorname{rmin}\left\{\widetilde{\mu_{F}}(x), \widetilde{\delta}\right\}$ for all $x \in X$.

Proof. Assume that $F \subseteq \vee q_{(\widetilde{\gamma}, \widetilde{\delta})} G$. Let $x \in X$. If $\operatorname{rmax}\left\{\widetilde{\mu_{G}}(x), \tilde{\gamma}\right\}<\operatorname{rmin}\left\{\widetilde{\mu_{F}}(x), \widetilde{\delta}\right\}$, then there exists $\tilde{r}$ such that $\operatorname{rmax}\left\{\widetilde{\mu_{G}}(x), \tilde{\gamma}\right\}<\widetilde{r}<\operatorname{rmin}\left\{\widetilde{\mu_{F}}(x), \widetilde{\delta}\right\}$; that is, $U(x ; \widetilde{r}) \epsilon_{\tilde{\gamma}} F$ but $U(x ; \widetilde{r}) \overline{\epsilon_{\tilde{\gamma}} \vee q_{\tilde{\delta}}} G$, a contradiction. Hence $\operatorname{rmax}\left\{\widetilde{\mu_{G}}(x), \widetilde{\gamma}\right\} \geq \operatorname{rmin}\left\{\widetilde{\mu_{F}}(x), \widetilde{\delta}\right\}$.

Conversely, assume that $\operatorname{rmax}\left\{\widetilde{\mu_{G}}(x), \widetilde{\gamma}\right\} \geq \operatorname{rmin}\left\{\widetilde{\mu_{F}}(x), \widetilde{\delta}\right\}$ for all $x \in X$. If $F \overline{\subseteq \vee q_{(\tilde{\gamma}, \tilde{\delta})}} G$, then there exist $x \in X$ and $\tilde{r}>\tilde{\gamma}$ such that $U(x ; \widetilde{r}) \epsilon_{\tilde{\gamma}} F$ but $U(x ; \widetilde{r}) \overline{\epsilon_{\tilde{\gamma}} \vee q_{\tilde{\delta}}} G$, and so $\widetilde{\mu_{F}}(x) \geq \tilde{r}, \widetilde{\mu_{G}}(x)<\tilde{r}$, and $\widetilde{\mu_{G}}(x)+\widetilde{r}<2 \widetilde{\delta}$. This gives that $\operatorname{rmax}\left\{\widetilde{\mu_{G}}(x), \widetilde{\gamma}\right\}<\operatorname{rmin}\left\{\widetilde{\mu_{F}}(x), \widetilde{\delta}\right\}$, a contradiction. Hence $F \subseteq$ $\vee q_{(\tilde{\gamma}, \widetilde{\delta})} G$.

Lemma 3. Let $X$ be a nonempty set and $F, G, H \in \mathbb{E}(X)$ such that $F \subseteq \vee q_{(\tilde{\gamma}, \tilde{\delta})} G \subseteq \vee q_{(\tilde{\gamma}, \tilde{\delta})} H$. Then $F \subseteq \vee q_{(\widetilde{\gamma}, \widetilde{\delta})} H$.

Proof. It is straightforward by Lemma 2.
Note that Lemma 2 gives that $F \approx G$ if and only if $\operatorname{rmax}\left\{\operatorname{rmin}\left\{\widetilde{\mu_{F}}(x), \widetilde{\delta}\right\}, \widetilde{\gamma}\right\}=\operatorname{rmax}\left\{\operatorname{rmin}\left\{\widetilde{\mu_{G}}(x), \widetilde{\delta}\right\}, \widetilde{\gamma}\right\}$ for all $x \in X$ and $F, G \in \mathbb{E}(X)$, and it follows from Lemmas 2 and 3 that " $\approx$ " is an equivalence relation on $\mathbb{R}(X)$.

We now define some operations of interval valued fuzzy subsets of $R$.

Definition 4. Let $F, G \in \mathbb{F}(R)$. Define the sum and product of $F$ and $G$, denoted by $F \oplus G$ and $F \otimes G$, by

$$
\begin{gathered}
\widetilde{\mu_{F \oplus G}}(x)=\bigvee_{x \in y+z} \operatorname{rmin}\left\{\widetilde{\mu_{F}}(y), \widetilde{\mu_{G}}(z)\right\}, \\
\widetilde{\mu_{F \otimes G}}(x)=\left\{\begin{array}{c}
\bigvee_{x=y z} \operatorname{rmin}\left\{\widetilde{\mu_{F}}(y), \widetilde{\mu_{G}}(z)\right\} \\
\text { if there exist } y, z \in R \text { such that } x=y z \\
{[0,0]} \\
\text { otherwise, }
\end{array}\right.
\end{gathered}
$$

for all $x \in R$.

By Lemma 2 and Definition 4, we can easily deduce the following results.

Lemma 5. Let $F_{1}, F_{2}, G_{1}, G_{2} \in \mathbb{E}(R)$ be such that $F_{1} \subseteq$ $\vee q_{(\tilde{\gamma}, \tilde{\delta})} F_{2}$ and $G_{1} \subseteq \vee q_{(\tilde{\gamma}, \tilde{\delta})} G_{2}$. Then

(1) $F_{1} \oplus G_{1} \subseteq \vee q_{(\tilde{\gamma}, \widetilde{\delta})} F_{2} \oplus G_{2}, F_{1} \otimes G_{1} \subseteq \vee q_{(\tilde{\gamma}, \widetilde{\delta})} F_{2} \otimes G_{2}$;

(2) $F_{1} \cap G_{1} \subseteq \vee q_{(\widetilde{\gamma}, \widetilde{\delta})} F_{2} \cap G_{2}$.

Lemma 6. Let $F, G, H \in \mathbb{E}(R)$. Consider

(1) $F \oplus G=G \oplus F$;

(2) $F \oplus(G \oplus H)=(F \oplus G) \oplus H, F \otimes(G \otimes H)=(F \otimes G) \otimes H$;

(3) $F \oplus(G \cup H)=F \oplus G \cup F \oplus H, F \otimes(G \cup H)=F \otimes G \cup F \otimes H$;

(4) $F \oplus(G \cap H) \subseteq F \oplus G \cap F \oplus H, F \otimes(G \cap H) \subseteq F \otimes G \cap F \otimes H$.

Lemma 5 indicates that the equivalence relation " $\approx$ " is a congruence relation on $(\mathbb{V}(R), \oplus)$.

\section{Generalized Interval Valued $(\alpha, \beta)$-Fuzzy Hyperideals}

In this section, we define and investigate generalized interval valued $(\alpha, \beta)$-fuzzy hyperideals of a hyperring, where $\alpha \epsilon$ $\left\{\epsilon_{\tilde{\gamma}}, q_{\tilde{\delta}}, \epsilon_{\tilde{\gamma}} \vee q_{\tilde{\delta}}\right\}$ and $\beta \in\left\{\epsilon_{\tilde{\gamma}}, q_{\tilde{\delta}}, \epsilon_{\tilde{\gamma}} \wedge q_{\tilde{\delta}}, \epsilon_{\tilde{\gamma}} \vee q_{\tilde{\delta}}\right\}$.

Definition 7. An interval valued fuzzy subset $F$ of $R$ is called an interval valued ( $\alpha, \beta)$-fuzzy hyperideal of $R$ if, for all $\tilde{\gamma}<$ $\tilde{r} \leq[1,1], \tilde{\gamma}<\tilde{t} \leq[1,1]$, and $x, y \in R$ :

(Fla) $U(x ; \widetilde{r}) \alpha F$ and $U(y ; \widetilde{t}) \alpha F \Rightarrow U(z ; \operatorname{rmin}\{\tilde{r}, \tilde{t}\}) \beta F$ for all $z \in x+y$;

(F2a) $U(x ; \widetilde{r}) \alpha F \Rightarrow U(-x ; \widetilde{r}) \beta F$ for all $x \in R$;

(F3a) $U(x ; \widetilde{r}) \alpha F$ and $U(y ; \tilde{t}) \alpha F \Rightarrow U(x y ; \operatorname{rmin}\{\tilde{r}, \tilde{t}\})$ $\beta F$ for all $x, y \in R$. 
An interval valued $(\alpha, \beta)$-fuzzy hyperideal with $\tilde{\gamma}=[0,0]$ and $\widetilde{\delta}=[0.5,0.5]$ is called an interval valued $(\alpha, \beta)$-fuzzy hyperideal studied in [21].

The case $\alpha=\epsilon_{\gamma} \wedge q_{\delta}$ must be omitted since for an interval valued fuzzy subset $F$ of $R$ such that $\widetilde{\mu_{F}}(x) \leq \widetilde{\delta}$ for any $x \in R$ in the case $U(x ; \widetilde{r}) \in \wedge q F$ we have $\widetilde{\mu_{F}}(x) \geq \tilde{r}$ and $\widetilde{\mu_{F}}(x)+\tilde{r}>2 \tilde{\delta}$. Thus $\widetilde{\mu_{F}}(x)+\widetilde{\mu_{F}}(x)>\widetilde{\mu_{F}}(x)+\tilde{r}>2 \tilde{\delta}$, which implies $\widetilde{\mu_{F}}(x)>\widetilde{\delta}$. This means that $\{U(x ; \widetilde{r}): U(x ; \widetilde{r}) \in$ $\wedge q F\}=\emptyset$.

As it is not difficult to see, each interval valued $(\alpha, \beta)$ fuzzy hyperideal of $R$ is an interval valued $\left(\alpha, \epsilon_{\tilde{\gamma}} \vee q_{\tilde{\delta}}\right)$ fuzzy hyperideal of $R$. So, in the theory of interval valued $(\alpha, \beta)$-fuzzy hyperideals the central role is played by interval valued $\left(\alpha, \epsilon_{\tilde{\gamma}} \vee q_{\tilde{\delta}}\right)$-fuzzy hyperideals and we only need to investigate the properties of interval valued $\left(\alpha, \epsilon_{\tilde{\gamma}} \vee q_{\tilde{\delta}}\right)$ fuzzy hyperideals. In what follows, let $\alpha \in\left\{\epsilon_{\tilde{\gamma}}, q_{\tilde{\delta}}, \epsilon_{\tilde{\gamma}} \vee q_{\tilde{\delta}}\right\}$ unless otherwise specified.

Example 8. Let $R=\{a, b, c, d\}$ be a set with a hyperoperation "+" and a binary operation "." as follows:

\begin{tabular}{c|cccc}
+ & $a$ & $b$ & $c$ & $d$ \\
\hline$a$ & $\{a\}$ & $\{b\}$ & $\{c\}$ & $\{d\}$ \\
$b$ & $\{b\}$ & $\{a, b\}$ & $\{d\}$ & $\{c\}$ \\
$c$ & $\{c\}$ & $\{d\}$ & $\{a, c\}$ & $\{b\}$ \\
$d$ & $\{d\}$ & $\{c\}$ & $\{b\}$ & $\{a, d\}$, \\
$\cdot$ & $a$ & $b$ & $c$ & $d$ \\
\hline$a$ & $\{a\}$ & $\{a\}$ & $\{a\}$ & $\{a\}$ \\
$b$ & $\{a\}$ & $\{b\}$ & $\{b\}$ & $\{b\}$ \\
$c$ & $\{a\}$ & $\{c\}$ & $\{c\}$ & $\{c\}$ \\
$d$ & $\{a\}$ & $\{d\}$ & $\{d\}$ & $\{d\}$.
\end{tabular}

Then $(R,+, \cdot)$ is a hyperring $([21])$. Define an interval valued fuzzy subset $F$ of $R$ by

$$
\begin{array}{ll}
\widetilde{\mu_{F}}(a)=[0.6,0.7], & \widetilde{\mu_{F}}(b)=[0.2,0.4], \\
\widetilde{\mu_{F}}(c)=[0.2,0.4], & \widetilde{\mu_{F}}(d)=[0.2,0.4] .
\end{array}
$$

Then $F$ is an interval valued $\left(\alpha, \epsilon_{[0.2,0.4]} \vee q_{[0.6,0.7]}\right)$-fuzzy hyperideal of $R$.

Proposition 9. An interval valued $\left(\epsilon_{\tilde{\gamma}} \vee q_{\tilde{\delta}}, \epsilon_{\tilde{\gamma}} \vee q_{\tilde{\delta}}\right)$-fuzzy hyperideal $F$ of $R$ is an interval valued $\left(\epsilon_{\tilde{\gamma}}, \epsilon_{\tilde{\gamma}} \vee q_{\tilde{\delta}}\right)$-fuzzy hyperideal of $R$.

Proof. This is straightforward since $U(x ; \widetilde{r}) \epsilon_{\tilde{\gamma}} F$ implies $U(x ; \widetilde{r}) \epsilon_{\tilde{\gamma}} \vee q_{\tilde{\delta}} F$ for all $x \in R$ and $\tilde{\gamma}<\tilde{r} \leq[1,1]$.

Proposition 10. Any interval valued $\left(\epsilon_{\tilde{\gamma}}, \epsilon_{\tilde{\gamma}}\right)$-fuzzy hyperideal F of $R$ is an interval valued $\left(q_{\tilde{\delta}}, \epsilon_{\tilde{\gamma}} \vee q_{\tilde{\delta}}\right)$-fuzzy hyperideal of $R$.

Proof. This part is straightforward.

The following example shows that the converse of Propositions 9 and 10 may not be true in general.
Example 11. Consider Example 8. Define an interval valued fuzzy subset $F$ of $R$ by

$$
\begin{array}{ll}
\widetilde{\mu_{F}}(a)=[0.6,0.7], & \widetilde{\mu_{F}}(b)=[0.7,0.8], \\
\widetilde{\mu_{F}}(c)=[0.3,0.5], & \widetilde{\mu_{F}}(d)=[0.2,0.4] .
\end{array}
$$

Then

(1) it is routine to verify that $F$ is an $\left(\epsilon_{[0.3,0.5]} ; \epsilon_{[0.3,0.5]} \mathrm{V}\right.$ $\left.q_{[0.6,0.7]}\right)$-fuzzy hyperideal of $R$;

(2) $F$ is not an $\left(\epsilon_{[0.3,0.5]} ; \epsilon_{[0.3,0.5]}\right)$-fuzzy hyperideal of $R$ since $U(b ;[0.7,0.8]) \epsilon_{[0.3,0.5]} F$ but $a \in b+b$ and $U(a ;[0.7,0.8]) \overline{\epsilon_{[0.7,0.8]}} F$;

(3) $F$ is not an $\left(\epsilon_{[0.3,0.5]} \vee q_{[0.6,0.7]} ; \epsilon_{[0.3,0.5]} \vee q_{[0.6,0.7]}\right)$ fuzzy hyperideal of $R$ since $U(b ;[1,1]) \epsilon_{[0.3,0.5]} \vee$ $q_{[0.6,0.7]} F$ and $U(c ;[1,1]) \epsilon_{[0.3,0.5]} \vee q_{[0.6,0.7]} F$ but

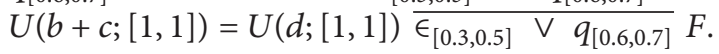

Theorem 12. Let $2 \widetilde{\delta}=[1,1]+\widetilde{\gamma}$ and $F$ be an interval valued $\left(\alpha, \epsilon_{\tilde{\gamma}} \vee q \tilde{\delta}\right)$-fuzzy hyperideal of $R$. Then the set $F_{\tilde{\tilde{\gamma}}}$ is a hyperideal of $R$, where $F_{\widetilde{\gamma}}=\left\{x \in R \mid \widetilde{\mu_{F}}(x)>\widetilde{\gamma}\right\}$.

Proof. Assume that $F$ is an interval valued $\left(\alpha, \epsilon_{\tilde{\gamma}} \vee q_{\tilde{\delta}}\right)$-fuzzy hyperideal of $R$. Let $x, y \in F_{\tilde{\tilde{\gamma}}}$. Then $\widetilde{\mu_{F}}(x)>\tilde{\gamma}, \widetilde{\mu_{F}}(y)>\widetilde{\gamma}$. We consider the following two cases.

Case 1. If $\alpha \in\left\{\epsilon_{\tilde{\gamma}}, \epsilon_{\tilde{\gamma}} \vee q_{\tilde{\delta}}\right\}$, then $U\left(x ; \widetilde{\mu_{F}}(x)\right) \alpha F$ and $U\left(y ; \widetilde{\mu_{F}}(y)\right) \alpha F$. By (Fla), $U\left(z ; \operatorname{rmin}\left\{\widetilde{\mu_{F}}(x), \widetilde{\mu_{F}}(y)\right\}\right) \epsilon_{\tilde{\gamma}} \vee$ $q_{\widetilde{\delta}} F$ for all $z \in x+y$, that is, $U\left(z ; \operatorname{rmin}\left\{\widetilde{\mu_{F}}(x), \widetilde{\mu_{F}}(y)\right\}\right) \epsilon_{\tilde{\gamma}} F$ or $U\left(z ; \operatorname{rmin}\left\{\widetilde{\mu_{F}}(x), \widetilde{\mu_{F}}(y)\right\}\right) q_{\tilde{\delta}} F$. It follows that $F(z) \geq$ $\operatorname{rmin}\left\{\widetilde{\mu_{F}}(x), \widetilde{\mu_{F}}(y)\right\}>\widetilde{\gamma}$ or $\widetilde{\mu_{F}}(z)+\operatorname{rmin}\left\{\widetilde{\mu_{F}}(x), \widetilde{\mu_{F}}(y)\right\}>2 \widetilde{\delta}$, and so $\widetilde{\mu_{F}}(z) \geq \operatorname{rmin}\left\{\widetilde{\mu_{F}}(x), \widetilde{\mu_{F}}(y)\right\}>\widetilde{\gamma}$ or $\widetilde{\mu_{F}}(z)>2 \widetilde{\delta}-$ $\operatorname{rmin}\left\{\widetilde{\mu_{F}}(x), \widetilde{\mu_{F}}(y)\right\} \geq 2 \widetilde{\delta}-[1,1]=\tilde{\gamma}$. Hence $\widetilde{\mu_{F}}(z)>\tilde{\gamma}$ and so $z \in F_{\widehat{\tilde{\gamma}}}$. Therefore, $x+y \subseteq F_{\tilde{\gamma}}$.

By $(\mathrm{F} 2 \mathrm{a})$ and $(\mathrm{F} 3 \mathrm{a}), U\left(-x ; \widetilde{\mu_{F}}(x)\right) \epsilon_{\tilde{\gamma}} \vee q_{\tilde{\delta}} F$ and $U\left(x y ; \operatorname{rmin}\left\{\widetilde{\mu_{F}}(x), \widetilde{\mu_{F}}(y)\right\}\right) \epsilon_{\tilde{\gamma}} \vee q_{\widetilde{\delta}} F$. Analogous to the above proof, we have $\widetilde{\mu_{F}}(x)>\widetilde{\gamma}$ and $\widetilde{\mu_{F}}(x y)>\tilde{\gamma}$; that is, $-x \in F_{\tilde{\gamma}}$ and $x y \in F_{\tilde{\gamma}}$. Therefore, $F_{\widetilde{\gamma}}$ is a hyperideal of $R$.

Case 2. If $\alpha=q_{\tilde{\delta}}$, then $U(x ;[1,1]) \alpha F$ and $U(y ;[1,1]) \alpha F$ since $2 \widetilde{\delta}=[1,1]+\widetilde{\gamma}$. Analogous to the proof of Case 1 , we may prove that $F_{\tilde{\gamma}}$ is a hyperideal of $R$.

If we take $\widetilde{\gamma}=[0,0]$ and $\widetilde{\delta}=[0.5,0.5]$ in Theorem 12 , then we have the following corollary.

Corollary 13. Let $F$ be an interval valued ( $\alpha, \in \vee q)$-fuzzy hyperideal of $R$. Then the set $F_{\widehat{[0,0]}}$ is a hyperideal of $R$, where $\alpha \in\{\in, q, \in \vee q\}$.

Theorem 14. Let $2 \widetilde{\delta}=[1,1]+\widetilde{\gamma}$ and $A$ be a nonempty subset of $R$. Then $A$ is a hyperideal of $R$ if and only if the interval valued fuzzy subset $F$ of $R$ such that $\widetilde{\mu_{F}}(x) \geq \widetilde{\delta}$ for all $x \in A$ and $\widetilde{\mu_{F}}(x) \leq \tilde{\gamma}$ otherwise is an interval valued $\left(\alpha, \epsilon_{\tilde{\gamma}} \vee q_{\tilde{\delta}}\right)$-fuzzy hyperideal of $R$. 
Proof. Assume that $A$ is a hyperideal of $R$. Let $x, y \in R$ and $\widetilde{\gamma}<\widetilde{r} \leq[1,1], \widetilde{\gamma}<\widetilde{t} \leq[1,1]$ be such that $U(x ; \widetilde{r}) \propto F$ and $U(y ; \tilde{t}) \propto F$. Then we have the following four cases.

Case 1. If $U(x ; \widetilde{r}) \epsilon_{\tilde{\gamma}} F$ and $U(y ; \tilde{t}) \epsilon_{\tilde{\gamma}} F$, then $\widetilde{\mu_{F}}(x) \geq \tilde{r}>\tilde{\gamma}$ and $\widetilde{\mu_{F}}(y) \geq \widetilde{t}>\tilde{\gamma}$. Thus, $\widetilde{\mu_{F}}(x) \geq \widetilde{\delta}$ and $\widetilde{\mu_{F}}(y) \geq \widetilde{\delta}$; that is, $x, y \in A$.

Case 2. If $U(x ; \widetilde{r}) q_{\tilde{\delta}} F$ and $U(y ; \widetilde{t}) q_{\tilde{\delta}} F$, then $\widetilde{\mu_{F}}(x)+\widetilde{r}>2 \widetilde{\delta}$ and $\widetilde{\mu_{F}}(y)+\widetilde{t}>2 \widetilde{\delta}$, and so $\widetilde{\mu_{F}}(x)>2 \widetilde{\delta}-\tilde{r} \geq 2 \widetilde{\delta}-[1,1]=\tilde{\gamma}$ and $\widetilde{\mu_{F}}(y)>2 \widetilde{\delta}-\widetilde{t} \geq 2 \widetilde{\delta}-[1,1]=\widetilde{\gamma}$. It follows that $\widetilde{\mu_{F}}(x) \geq \widetilde{\delta}$ and $\widetilde{\mu_{F}}(y) \geq \widetilde{\delta}$; that is, $x, y \in A$.

Case 3. If $U(x ; \widetilde{r}) \epsilon_{\tilde{\gamma}} F$ and $U(y ; \widetilde{t}) q_{\tilde{\delta}} F$, then $\widetilde{\mu_{F}}(x) \geq \widetilde{r}>\tilde{\gamma}$ and $\widetilde{\mu_{F}}(y)+\widetilde{t}>2 \widetilde{\delta}$. Analogous to the proof of Cases 1 and 2 , $\widetilde{\mu_{F}}(x) \geq \widetilde{\delta}$ and $\widetilde{\mu_{F}}(y) \geq \widetilde{\delta}$; that is, $x, y \in A$.

Case 4. If $U(x ; \widetilde{r}) q_{\widetilde{\delta}} F$ and $U(y ; \widetilde{t}) \epsilon_{\tilde{\gamma}} F$, then $\widetilde{\mu_{F}}(x)+\widetilde{r}>2 \widetilde{\delta}$ and $\widetilde{\mu_{F}}(y) \geq \widetilde{t}>\tilde{\gamma}$. Analogous to the proof of Cases 1 and 2 , $\widetilde{\mu_{F}}(x) \geq \widetilde{\delta}$ and $\widetilde{\mu_{F}}(y) \geq \widetilde{\delta}$; that is, $x, y \in A$.

Thus, in any case, $x, y \in A$. Hence $z \in A$ for all $z \in x+y$, which implies that $\widetilde{\mu_{F}}(z) \geq \widetilde{\delta}$. If $\operatorname{rmin}\left\{\widetilde{\mu_{F}}(x), \widetilde{\mu_{F}}(y)\right\} \leq \widetilde{\delta}$, then $\widetilde{\mu_{F}}(z) \geq \widetilde{\delta} \geq \operatorname{rmin}\left\{\widetilde{\mu_{F}}(x), \widetilde{\mu_{F}}(y)\right\}>\widetilde{\gamma}$; it follows that $U\left(z ; \operatorname{rmin}\left\{\widetilde{\mu_{F}}(x), \widetilde{\mu_{F}}(y)\right\}\right) \epsilon_{\tilde{\gamma}} F$. If $\operatorname{rmin}\left\{\widetilde{\mu_{F}}(x), \widetilde{\mu_{F}}(y)\right\}>$ $\widetilde{\delta}$, then $\widetilde{\mu_{F}}(z)+\operatorname{rmin}\left\{\widetilde{\mu_{F}}(x), \widetilde{\mu_{F}}(y)\right\}>\widetilde{\delta}+\widetilde{\delta}=$ $2 \widetilde{\delta}$; it follows that $U\left(z ; \operatorname{rmin}\left\{\widetilde{\mu_{F}}(x), \widetilde{\mu_{F}}(y)\right\}\right) q_{\widetilde{\delta}} f$. Therefore, $U\left(z ; \operatorname{rmin}\left\{\widetilde{\mu_{F}}(x), \widetilde{\mu_{F}}(y)\right\}\right) \epsilon_{\tilde{\gamma}} \vee q_{\tilde{\delta}} f$. In a similar way, we may prove that $U\left(-x ; \widetilde{\mu}_{F}(x)\right) \epsilon_{\tilde{\gamma}} \vee q_{\widetilde{\delta}} F$ and $U\left(x y ; \operatorname{rmin}\left\{\widetilde{\mu_{F}}(x), \widetilde{\mu_{F}}(y)\right\}\right) \epsilon_{\tilde{\gamma}} \vee q_{\tilde{\delta}} F$. Therefore, $F$ is an interval valued $\left(\alpha, \epsilon_{\tilde{\gamma}} \vee q_{\tilde{\delta}}\right)$-fuzzy hyperideal of $R$.

Conversely, assume that $F$ is an interval valued $\left(\alpha, \epsilon_{\tilde{\gamma}} \vee\right.$ $\left.q_{\tilde{\delta}}\right)$-fuzzy hyperideal of $R$. It is easy to see that $A=F_{\tilde{\gamma}}$. It follows from Theorem 12 that $A$ is a hyperideal of $R$.

If we take $\widetilde{\gamma}=[0,0]$ and $\widetilde{\delta}=[0.5,0.5]$ in Theorem 14 , then we have the following corollary.

Corollary 15. Let $A$ be a nonempty subset of $R$. Then $A$ is a hyperideal of $R$ if and only if the interval valued fuzzy subset $F$ of $R$ such that $\widetilde{\mu_{F}}(x) \geq[0.5,0.5]$ for all $x \in A$ and $\widetilde{\mu_{F}}(x)=$ $[0,0]$ otherwise is an $(\alpha, \in \vee q)$-fuzzy hyperideal of $R$, where $\alpha \in\{\epsilon, q, \in \vee q\}$.

Theorem 16. Let $F$ be an $\left(q_{\tilde{\delta}}, \epsilon_{\tilde{\gamma}} \vee q_{\tilde{\delta}}\right)$-fuzzy hyperideal of $R$. Then the following conditions hold:

(F1b) $\operatorname{rmax}\left\{\bigwedge_{z \in x+y} \widetilde{\mu_{F}}(z), \widetilde{\gamma}\right\} \geq \operatorname{rmin}\left\{\widetilde{\mu_{F}}(x), \widetilde{\mu_{F}}(y), \widetilde{\delta}\right\}$ for all $x, y \in R$;

(F2b) $\operatorname{rmax}\left\{\widetilde{\mu_{F}}(-x), \widetilde{\gamma}\right\} \geq \operatorname{rmin}\left\{\widetilde{\mu_{F}}(x), \widetilde{\delta}\right\}$ for all $x \in R$;

(F3b) $\operatorname{rmax}\left\{\widetilde{\mu_{F}}(x y), \widetilde{\gamma}\right\} \geq \operatorname{rmin}\left\{\widetilde{\mu_{F}}(x), \widetilde{\mu_{F}}(y), \widetilde{\delta}\right\}$ for all $x \in$ $R$.

Proof. Let $F$ be an $\left(q_{\widetilde{\delta}}, \epsilon_{\tilde{\gamma}} \vee q_{\tilde{\delta}}\right)$-fuzzy hyperideal of $R$. We only show (F1b). The other properties can be similarly proved. Assume that there exist $x, y, z \in R$ such that $z \in x+y$ and $\operatorname{rmax}\left\{\widetilde{\mu_{F}}(z), \tilde{\gamma}\right\}<\operatorname{rmin}\left\{\widetilde{\mu_{F}}(x), \widetilde{\mu_{F}}(y), \widetilde{\delta}\right\}$. Then, for all $\tilde{r} \in D[0,1]$ such that $2 \widetilde{\delta}-\operatorname{rmax}\left\{\widetilde{\mu_{F}}(z), \tilde{\gamma}\right\}>\widetilde{r}>2 \widetilde{\delta}-$ $\operatorname{rmin}\left\{\widetilde{\mu_{F}}(x), \widetilde{\mu_{F}}(y), \widetilde{\delta}\right\}$, we have

$$
\begin{aligned}
2 \widetilde{\delta}-\widetilde{\mu_{F}}(z) & \geq 2 \widetilde{\delta}-\operatorname{rmax}\left\{\widetilde{\mu_{F}}(z), \widetilde{\gamma}\right\}>\widetilde{r} \\
& >\operatorname{rmax}\left\{2 \widetilde{\delta}-\widetilde{\mu_{F}}(x), 2 \widetilde{\delta}-\widetilde{\mu_{F}}(y), \widetilde{\delta}\right\},
\end{aligned}
$$

and so $\widetilde{\mu_{F}}(x)+\widetilde{r}>2 \widetilde{\delta}, \widetilde{\mu_{F}}(y)+\widetilde{r}>2 \tilde{\delta}, \widetilde{\mu_{F}}(z)+\widetilde{r}<$ $2 \tilde{\delta}$, and $\widetilde{\mu_{F}}(z)<\tilde{\delta}<\tilde{r}$. Hence $U(x ; \widetilde{r}) q_{\tilde{\delta}} F, U(y ; \widetilde{r}) q_{\tilde{\delta}} F$ but $U(z ; \widetilde{r}) \overline{\epsilon_{\tilde{\gamma}} \vee q_{\tilde{\delta}}} F$, a contradiction. Hence (F1b) is valid.

\section{Interval Valued $\left(\epsilon_{\tilde{\gamma}}, \epsilon_{\tilde{\gamma}} \vee q_{\tilde{\delta}}\right)$-Fuzzy Hyperideals}

In this section, using a new ordering relation on $\mathbb{R}(R)$, we investigate the interval valued $\left(\epsilon_{\tilde{\gamma}}, \epsilon_{\tilde{\gamma}} \vee q_{\tilde{\delta}}\right)$-fuzzy hyperideals of a hyperring. In what follows, we take $\alpha=\epsilon_{\tilde{\gamma}}$ and $\beta=\epsilon_{\tilde{\gamma}} \vee q_{\tilde{\delta}}$ in Definition 7. Before proceeding, we present some characterizations of interval valued $\left(\epsilon_{\tilde{\gamma}}, \epsilon_{\tilde{\gamma}} \vee q_{\tilde{\delta}}\right)$-fuzzy hyperideals.

Lemma 17. Let $F \in \mathbb{E}(H)$. Then (F1a) holds if and only if one of the following conditions holds:

(F1b) $\operatorname{rmax}\left\{\bigwedge_{z \in x+y} \widetilde{\mu_{F}}(z), \widetilde{\gamma}\right\} \geq \operatorname{rmin}\left\{\widetilde{\mu_{F}}(x), \widetilde{\mu_{F}}(y), \widetilde{\delta}\right\}$ for all $x, y \in R$;

(F1c) $F \oplus F \subseteq \vee q_{(\tilde{\gamma}, \widetilde{\delta})} F$.

Proof. (F1a) $\Rightarrow(\mathrm{F} 1 \mathrm{~b})$ Let $x, y \in R$. Let, if possible, $z \in$ $R$ be such that $z \in x+y$ and $\operatorname{rmax}\left\{\widetilde{\mu_{F}}(z), \widetilde{\gamma}\right\}<$ $\widetilde{r}=\operatorname{rmin}\left\{\widetilde{\mu_{F}}(x), \widetilde{\mu_{F}}(y), \widetilde{\delta}\right\}$. Then $U(x ; \widetilde{r}) \epsilon_{\tilde{\gamma}} F, U(y ; \widetilde{r}) \epsilon_{\tilde{\gamma}} F$, $U(z ; \widetilde{r}) \overline{\epsilon_{\tilde{\gamma}}} F$, and $\widetilde{\mu_{F}}(z)+\widetilde{r}<\tilde{r}+\widetilde{r} \leq 2 \widetilde{\delta}$; that is, $U(z ; \widetilde{r}) \frac{\epsilon_{\tilde{\gamma}} \vee q_{\tilde{\delta}}}{F}$, a contradiction. Hence (F1b) is valid.

$(\mathrm{F} 1 \mathrm{~b}) \Rightarrow(\mathrm{F} 1 \mathrm{c})$ For $U(x ; \widetilde{r}) \in_{\tilde{\gamma}} F \oplus F$, suppose, if possible, $U(x ; \widetilde{r}) \overline{\epsilon_{\tilde{\gamma}} \vee q_{\tilde{\delta}}} F$. Then $U(x ; \widetilde{r}) \overline{\epsilon_{\tilde{\gamma}}} F$ and $U(x ; \widetilde{r}) \overline{q_{\tilde{\delta}}} F$; that is, $\widetilde{\mu_{F}}(x)<\tilde{r}$ and $\widetilde{\mu_{F}}(x)+\widetilde{r} \leq 2 \widetilde{\delta}$ which implies that $\widetilde{\mu_{F}}(x)<\widetilde{\delta}$. If $x \in y+z$ for some $y, z \in R$, by (F1b), we have $\widetilde{\delta}>\operatorname{rmax}\left\{\widetilde{\mu_{F}}(x), \widetilde{\gamma}\right\} \geq \operatorname{rmin}\left\{\widetilde{\mu_{F}}(y), \widetilde{\mu_{F}}(z), \widetilde{\delta}\right\}$, it follows that $\operatorname{rmax}\left\{\widetilde{\mu_{F}}(x), \widetilde{\gamma}\right\} \geq \operatorname{rmin}\left\{\widetilde{\mu_{F}}(y), \widetilde{\mu_{F}}(z)\right\}$. Hence we have

$$
\begin{aligned}
\tilde{r} & \leq(F \oplus F)(x)=\bigvee_{x \in a+b} \operatorname{rmin}\left\{\widetilde{\mu_{F}}(y), \widetilde{\mu_{F}}(z)\right\} \\
& \leq \bigvee_{x \in a+b} \operatorname{rmax}\left\{\widetilde{\mu_{F}}(x), \tilde{\gamma}\right\}=\operatorname{rmax}\left\{\widetilde{\mu_{F}}(x), \tilde{\gamma}\right\} ;
\end{aligned}
$$

which contradicts $\tilde{\gamma}<\tilde{r}$ and $\widetilde{\mu_{F}}(x)<\widetilde{r}$. Hence (F1c) is satisfied.

(F1c) $\Rightarrow$ (F1a) Let $x, y \in R$ and $\tilde{\gamma}<\tilde{r} \leq[1,1], \tilde{\gamma}<\tilde{t} \leq[1,1]$ be such that $U(x ; \widetilde{r}) \epsilon_{\tilde{\gamma}} F$ and $U(y ; \tilde{t}) \epsilon_{\tilde{\gamma}} F$. Then for any $z \in$ $x+y$, we have

$$
\begin{aligned}
(F \oplus F)(z) & =\bigvee_{z \in a+b} \operatorname{rmin}\left\{\widetilde{\mu_{F}}(a), \widetilde{\mu_{F}}(b)\right\} \\
& \geq \operatorname{rmin}\left\{\widetilde{\mu_{F}}(x), \widetilde{\mu_{F}}(y)\right\} \geq \operatorname{rmin}\{\widetilde{r}, \widetilde{t}\}>\widetilde{\gamma}
\end{aligned}
$$

Hence $U\left(z ; \operatorname{rmin}\left\{\widetilde{\mu_{F}}(x), \widetilde{\mu_{F}}(y)\right\}\right) \epsilon_{\tilde{\gamma}} F \oplus F$ and so (F1a) holds. 
Lemma 18. Let $F \in \mathbb{E}(R)$. Then (F2a) holds if and only if one of the following conditions holds:

(F2b) $\operatorname{rmax}\left\{\widetilde{\mu_{F}}(-x), \tilde{\gamma}\right\} \geq \operatorname{rmin}\left\{\widetilde{\mu_{F}}(x), \widetilde{\delta}\right\}$ for all $x \in R$

(F2c) $F \subseteq \vee q_{(\tilde{\gamma}, \tilde{\delta})}-F$, where $-F$ is defined by $\widetilde{\mu_{-F}}(x)=$ $\widetilde{\mu_{F}}(-x)$ for all $x \in R$.

Proof. The proof is analogous to the proof of Lemma 17.

Lemma 19. Let $F \in \mathbb{E}(R)$. Then (F3a) holds if and only if one of the following conditions holds:

(F3b) $\operatorname{rmax}\left\{\widetilde{\mu_{F}}(x y), \widetilde{\gamma}\right\} \geq \operatorname{rmin}\left\{\widetilde{\mu_{F}}(x), \widetilde{\mu_{F}}(y), \widetilde{\delta}\right\}$ for all $x \in$ $R$;

$(\mathrm{F} 3 \mathrm{c}) F \otimes F \subseteq \vee q_{(\tilde{\gamma}, \tilde{\delta})} F$.

Proof. The proof is analogous to Lemma 17.

From Lemmas 17-19, we obtain the following results.

Theorem 20. Let $F \in \mathbb{E}(R)$. Then $F$ is an $\left(\epsilon_{\tilde{\gamma}}, \epsilon_{\tilde{\gamma}} \vee q_{\tilde{\delta}}\right)$-fuzzy hyperideal of $R$ if and only if it satisfies conditions (F1b)-(F3b) or conditions (F1c) $-($ F3c).

Remark 21. For any interval valued $\left(\epsilon_{\tilde{\gamma}}, \epsilon_{\tilde{\gamma}} \vee q_{\tilde{\delta}}\right)$-fuzzy hyperideal $F$ of $R$, we can conclude that

(1) $F$ is an interval valued fuzzy hyperideal of $R$ when $\tilde{\gamma}=$ $[0,0]$ and $\widetilde{\delta}=[1,1]([21]$, Definition 4.1);

(2) $F$ is an interval valued $(\epsilon, \in \vee q)$-fuzzy hyperideal of $R$ when $\widetilde{\gamma}=[0,0]$ and $\widetilde{\delta}=[0.5,0.5]$ ([21], Definition 4.3);

(3) $F$ is an interval valued fuzzy hyperideal of $R$ with thresholds $(\widetilde{\gamma}, \widetilde{\delta})([21]$, Definition 4.9$)$.

Combining Theorems 16 and 20, we have the following result.

Proposition 22. Any interval valued $\left(q_{\tilde{\delta}}, \epsilon_{\tilde{\gamma}} \vee q_{\tilde{\delta}}\right)$-fuzzy hyperideal of $R$ is an interval valued $\left(\epsilon_{\tilde{\gamma}}, \epsilon_{\tilde{\gamma}} \vee q_{\tilde{\delta}}\right)$-fuzzy hyperideal of $R$.

The following example shows that the converse of Proposition 22 is not true in general.

Example 23. Let $R$ and $F$ be as in Example 11. Then $F$ is an $\left(\epsilon_{[0.3,0.5]} ; \epsilon_{[0.3,0.5]} \vee q_{[0.6,0.7]}\right)$-fuzzy hyperideal of $R$, but it is not an $\left(q_{[0.6,0.7]} ; \epsilon_{[0.3,0.5]} \vee q_{[0.6,0.7]}\right)$-fuzzy hyperideal of $R$ since $U(a ;[0.7,0.8]) q_{[0.6,0.7]} F$ and $U(c ;[1,1]) \epsilon_{[0.3,0.5]} \vee$ $q_{[0.6,0.7]} F$ but $U(a+c ; \operatorname{rmin}\{[0.7,0.8],[1,1]\})=$ $U(c ;[0.7,0.8]) \overline{\epsilon_{[0.3,0.5]} \vee q_{[0.6,0.7]}} F$.

If we take $\widetilde{\gamma}=[0,0]$ and $\widetilde{\delta}=[0.5,0.5]$ in Propositions 9, 10 , and 22 , then we have the following corollary.
Corollary 24. Let $F \in \mathbb{E}(R)$. Then

(1) any interval valued $(\epsilon \vee q, \in \vee q)$-fuzzy hyperideal of $R$ is an interval valued $(\epsilon, \in \vee q)$-fuzzy hyperideal of $R$ [21];

(2) any interval valued $(q, \in \vee q)$-fuzzy hyperideal of $R$ is an interval valued $(\epsilon, \in \vee q)$-fuzzy hyperideal of $R$;

(3) any interval valued $(\epsilon, \epsilon)$-fuzzy hyperideal of $R$ is an interval valued $(\in \vee q, \in \vee q)$-fuzzy hyperideal of $R$ [21].

For any $F \in \mathbb{E}(R)$, we define $F_{\widetilde{r}}=\left\{x \in R \mid U(x ; \widetilde{r}) \in_{\widetilde{\gamma}} F\right\}$, $F_{\widetilde{r}}^{\widetilde{\delta}}=\left\{x \in R \mid U(x ; \widetilde{r}) q_{\widetilde{\delta}} F\right\}$, and $[F]_{\widetilde{r}}^{\widetilde{\delta}}=\left\{x \in R \mid U(x ; \widetilde{r}) \epsilon_{\tilde{\gamma}} \vee\right.$ $\left.q_{\widetilde{\delta}} F\right\}$ for all $\widetilde{r} \in D^{\prime}[0,1]$. It is clear that $[F]_{\widetilde{r}}^{\widetilde{\delta}}=F_{\widetilde{r}} \cup F_{\widetilde{r}}^{\widetilde{\delta}}$.

The next theorem provides the relationship between the interval valued $\left(\epsilon_{\tilde{\gamma}}, \epsilon_{\tilde{\gamma}} \vee q_{\tilde{\delta}}\right)$-fuzzy hyperideals of $R$ and the crisp hyperideals of $R$.

Theorem 25. Let $F \in \mathbb{E}(R)$.

(1) $F$ is an interval valued $\left(\epsilon_{\tilde{\gamma}}, \epsilon_{\tilde{\gamma}} \vee q_{\tilde{\delta}}\right)$-fuzzy hyperideal of $R$ if and only if $F_{\widetilde{r}}\left(F_{\widetilde{r}} \neq \emptyset\right)$ is an hyperideal of $R$ for all $\tilde{\gamma}<\tilde{r} \leq \widetilde{\delta}$.

(2) If $2 \delta=1+\gamma$, then $F$ is an interval valued $\left(\epsilon_{\tilde{\gamma}}, \epsilon_{\tilde{\gamma}} \vee\right.$ $\left.q_{\tilde{\delta}}\right)$-fuzzy hyperideal of $R$ if and only if $F_{\widetilde{r}}^{\widetilde{\delta}}\left(F_{\widetilde{r}}^{\widetilde{\delta}} \neq \emptyset\right)$ is a hyperideal of $R$ for all $\widetilde{\delta}<\tilde{r} \leq[1,1]$.

(3) If $2 \delta=1+\gamma$, then $F$ is an interval valued $\left(\epsilon_{\tilde{\gamma}}, \epsilon_{\tilde{\gamma}} \vee q_{\tilde{\delta}}\right)$ fuzzy hyperideal of $R$ if and only if $[F]_{\widetilde{r}}^{\widetilde{\delta}}\left([F]_{\widetilde{r}}^{\widetilde{\delta}} \neq \emptyset\right)$ is a hyperideal of $R$ for all $\widetilde{\gamma}<\widetilde{r} \leq[1,1]$.

Proof. We only show (3). Let $F$ be an interval valued $\left(\epsilon_{\tilde{\gamma}}, \epsilon_{\tilde{\gamma}} \vee\right.$ $\left.q_{\tilde{\delta}}\right)$-fuzzy hyperideal of $R$ and $x, y \in[F]_{\tilde{r}}^{\tilde{\delta}}$ for some $\tilde{\gamma}<\tilde{r} \leq$ $[1,1]$. Then $U(x ; \widetilde{r}) \epsilon_{\tilde{\gamma}} \vee q_{\widetilde{\delta}} F$ and $U(y ; \widetilde{r}) \epsilon_{\tilde{\gamma}} \vee q_{\tilde{\delta}} F$; that is, $\widetilde{\mu_{F}}(x) \geq \widetilde{r}$ or $\widetilde{\mu_{F}}(x)>2 \widetilde{\delta}-\widetilde{r}>2 \widetilde{\delta}-[1,1]=\widetilde{\gamma}$, and $\widetilde{\mu_{F}}(y) \geq \tilde{r}$ or $\widetilde{\mu_{F}}(y)>2 \widetilde{\delta}-\tilde{r}>2 \widetilde{\delta}-[1,1]=\tilde{\gamma}$. Since $F$ is an interval valued $\left(\epsilon_{\tilde{\gamma}}, \epsilon_{\tilde{\gamma}} \vee q_{\tilde{\delta}}\right)$-fuzzy hyperideal of $R$, we have $\widetilde{\mu_{F}}(z) \geq \operatorname{rmin}\left\{\widetilde{\mu_{F}}(x), \widetilde{\mu_{F}}(y), \widetilde{\delta}\right\}$ for all $z \in x+y$ since $\tilde{\gamma}<\operatorname{rmin}\left\{\widetilde{\mu_{F}}(x), \widetilde{\mu_{F}}(y), \widetilde{\delta}\right\}$. We consider the following cases.

Case 1. Consider $\tilde{\gamma}<\tilde{r} \leq \widetilde{\delta}$. Since $\tilde{\gamma}<\tilde{r} \leq \widetilde{\delta}$, we have $2 \widetilde{\delta}-\tilde{r} \geq$ $\widetilde{\delta} \geq \widetilde{r}$ and so $\widetilde{\mu_{F}}(z) \geq \operatorname{rmin}\{\widetilde{r}, \widetilde{r}, \widetilde{\delta}\}=\widetilde{r}$ or $\widetilde{\mu_{F}}(z) \geq \operatorname{rmin}\{\widetilde{r}, 2 \widetilde{\delta}-$ $\widetilde{r}, \widetilde{\delta}\}=\widetilde{r}$, or $\widetilde{\mu_{F}}(z) \geq \operatorname{rmin}\{2 \widetilde{\delta}-\widetilde{r}, 2 \widetilde{\delta}-\widetilde{r}, \widetilde{\delta}\}=\widetilde{r}=\widetilde{\delta} \geq \widetilde{r}$. Hence $U(z ; \widetilde{r}) \epsilon_{\tilde{\gamma}} F$.

Case 2. Consider $\widetilde{\delta}<\tilde{r} \leq[1,1]$. Since $\widetilde{\delta}<\tilde{r} \leq[1,1]$, we have $2 \widetilde{\delta}-\widetilde{r}<\widetilde{\delta}<\widetilde{r}$ and so $\widetilde{\mu_{F}}(z) \geq \operatorname{rmin}\{\widetilde{r}, \widetilde{r}, \widetilde{\delta}\}=\widetilde{\delta}>2 \widetilde{\delta}-\widetilde{r}$ or $\widetilde{\mu_{F}}(z)>\operatorname{rmin}\{\widetilde{r}, 2 \widetilde{\delta}-\widetilde{r}, \widetilde{\delta}\}=2 \widetilde{\delta}-\widetilde{r}$, or $\widetilde{\mu_{F}}(z)>\operatorname{rmin}\{2 \widetilde{\delta}-$ $\widetilde{r}, 2 \widetilde{\delta}-\widetilde{r}, \widetilde{\delta}\}=2 \widetilde{\delta}-\widetilde{r}$. Hence $U(z ; \widetilde{r}) q_{\tilde{\delta}} F$.

Thus, in any case, $U(z ; \widetilde{r}) \epsilon_{\tilde{\gamma}} \vee q_{\tilde{\delta}} F$; that is, $z \in[F]_{\tilde{r}}^{\widetilde{\delta}}$ for all $z \in x+y$. Similarly we can show that $-x \in[F]_{\tilde{r}}^{\tilde{\delta}}$ and $x y \in[F]_{\tilde{r}}^{\widetilde{\delta}}$. Therefore, $[F]_{\widetilde{r}}^{\widetilde{\delta}}$ is a hyperideal of $R$.

Conversely, assume that the given condition holds. Let $x, y \in R$. If there exists $z \in R$ such that $z \in x+y$ and 
$\operatorname{rmax}\left\{\widetilde{\mu_{F}}(z), \widetilde{\gamma}\right\}<\widetilde{r}=\operatorname{rmin}\left\{\widetilde{\mu_{F}}(x), \widetilde{\mu_{F}}(y), \widetilde{\delta}\right\}$, then $x, y \in[F]_{\widetilde{r}}^{\widetilde{\delta}}$ but $z \notin[F]_{\tilde{r}}^{\tilde{\delta}}$, a contradiction. Therefore, $\operatorname{rmax}\left\{\widetilde{\mu_{F}}(z), \widetilde{\gamma}\right\} \geq$ $\operatorname{rmin}\left\{\widetilde{\mu_{F}}(x), \widetilde{\mu_{F}}(y), \widetilde{\delta}\right\}$ for all $z \in x+y$. Similarly we can show that conditions (F2b) and (F3b) are valid. Therefore, $F$ is an interval valued $\left(\epsilon_{\tilde{\gamma}}, \epsilon_{\tilde{\gamma}} \vee q_{\tilde{\delta}}\right)$-fuzzy hyperideal of $R$ by Theorem 20.

As a direct consequence of Theorem 25, we have the following result.

Corollary 26. Let $\widetilde{\gamma}, \widetilde{\gamma}^{\prime}, \widetilde{\delta}, \widetilde{\delta}^{\prime} \in D[0,1]$ be such that $\tilde{\gamma}<\widetilde{\delta}, \widetilde{\gamma}^{\prime}<$ $\widetilde{\delta}^{\prime}, \tilde{\gamma}<\widetilde{\gamma}^{\prime}$, and $\widetilde{\delta}^{\prime}<\widetilde{\delta}$. Then every interval valued $\left(\epsilon_{\tilde{\gamma}}, \epsilon_{\tilde{\gamma}} \vee\right.$ $\left.q_{\tilde{\delta}}\right)$-fuzzy hyperideal of $R$ is an interval valued $\left(\epsilon_{\tilde{\gamma}^{\prime}}, \epsilon_{\tilde{\gamma}^{\prime}} \vee q_{\tilde{\delta}^{\prime}}\right)$ fuzzy hyperideal of $R$.

The following example shows that the converse of Corollary 26 is not true in general.

Example 27. Consider Example 8. Define interval valued fuzzy subset $F$ of $R$ by

$$
\begin{array}{ll}
\widetilde{\mu_{F}}(a)=[0.6,0.7], \quad \widetilde{\mu_{F}}(b)=[0.2,0.3], \\
\widetilde{\mu_{F}}(c)=[0.3,0.4], \quad \widetilde{\mu_{F}}(d)=[0.3,0.4] .
\end{array}
$$

Then $F$ is an interval valued $\left(\epsilon_{[0.3,0.4]}, \epsilon_{[0.3,0.4]} \vee q_{[0.6,0.7]}\right)$ fuzzy hyperideal of $R$ but is not an interval valued $\left(\epsilon_{[0.2,0.3]}, \epsilon_{[0.2,0.3]} \vee q_{[0.6,0.7]}\right)$-fuzzy hyperideal of $R$.

If we take $\widetilde{\gamma}=[0,0]$ and $\widetilde{\delta}=[0.5,0.5]$ in Theorem 25, then we have the following corollary.

Corollary 28. Let $F \in \mathbb{R}(R)$.

(1) $F$ is an interval valued $(\epsilon, \in \vee q)$-fuzzy hyperideal of $R$ if and only if $F_{\vec{r}}\left(F_{\vec{r}} \neq \emptyset\right)$ is a hyperideal of $R$ for all $[0,0]<\widetilde{r} \leq[0.5,0.5]$.

(2) $F$ is an interval valued $(\epsilon, \in \vee q)$-fuzzy hyperideal of $R$ if and only if $F_{\tilde{r}}^{[0.5,0.5]}\left(F_{\tilde{r}}^{[0.5,0.5]} \neq \emptyset\right)$ is a hyperideal of $R$ for all $[0.5,0.5]<\tilde{r} \leq[1,1]$.

(3) $F$ is an interval valued $(\epsilon, \epsilon \vee q)$-fuzzy hyperideal of $R$ if and only if $[F]_{\tilde{r}}^{[0.5,0.5]}\left([F]_{\tilde{r}}^{[0.50 .5]} \neq \emptyset\right)$ is a hyperideal of $R$ for all $[0,0]<\widetilde{r} \leq[1,1]$.

Theorem 29. Let $F$ and $G$ be two interval valued $\left(\epsilon_{\tilde{\gamma}}, \epsilon_{\tilde{\gamma}} \vee\right.$ $\left.q_{\tilde{\delta}}\right)$-fuzzy hyperideals of $R$. Then so are $F \oplus G$ and $F \cap G$.

Proof. We only show that $F \oplus G$ is an interval valued $\left(\epsilon_{\tilde{\gamma}}, \epsilon_{\tilde{\gamma}} \vee\right.$ $q_{\tilde{\delta}}$ )-fuzzy hyperideal of $R$. The verification is as follows.

(1) By Lemmas 5, 6, and 17, $(F \oplus G) \oplus(F \oplus G)=(F \oplus F) \oplus$ $(G \oplus G) \subseteq \vee q_{(\widetilde{\gamma}, \widetilde{\delta})} F \oplus G$.
(2) Let $x \in R$. Then

$$
\begin{aligned}
\operatorname{rmax} & \left\{\widetilde{\mu_{F \oplus G}}(-x), \tilde{\gamma}\right\} \\
& =\operatorname{rmax}\left\{\bigvee_{-x \in y+z} \operatorname{rmin}\left\{\widetilde{\mu_{F}}(y), \widetilde{\mu_{G}}(z)\right\}, \widetilde{\gamma}\right\} \\
& =\bigvee_{-x \in y+z} \operatorname{rmin}\left\{\operatorname{rmax}\left\{\widetilde{\mu_{F}}(y), \widetilde{\gamma}\right\}, \operatorname{rmax}\left\{\widetilde{\mu_{G}}(z), \tilde{\gamma}\right\}\right\} \\
& =\bigvee_{x \in-y-z} \operatorname{rmin}\left\{\operatorname{rmax}\left\{\widetilde{\mu_{F}}(y), \widetilde{\gamma}\right\}, \operatorname{rmax}\left\{\widetilde{\mu_{G}}(z), \widetilde{\gamma}\right\}\right\} \\
& \geq \bigvee_{x \in-y-z} \operatorname{rmin}\left\{\operatorname{rmin}\left\{\widetilde{\mu_{F}}(-y), \widetilde{\delta}\right\}, \operatorname{rmin}\left\{\widetilde{\mu_{G}}(-z), \widetilde{\delta}\right\}\right\} \\
& =\operatorname{rmin}\left\{\bigvee_{x \in-y-z} \operatorname{rmin}\left\{\widetilde{\mu_{F}}(-y), \widetilde{\mu_{G}}(-z)\right\}, \widetilde{\delta}\right\} \\
& =\operatorname{rmin}\left\{\widetilde{\mu_{F \oplus G}}(x), \tilde{\delta}\right\} .
\end{aligned}
$$

(3) Let $x, y \in R$. We have

$$
\begin{aligned}
& \operatorname{rmin}\left\{\widetilde{\mu_{F \oplus G}}(x), \widetilde{\delta}\right\} \\
& =\operatorname{rmin}\left\{\bigvee_{x \in a+b} \operatorname{rmin}\left\{\widetilde{\mu_{F}}(a), \widetilde{\mu_{G}}(b)\right\}, \widetilde{\delta}\right\} \\
& =\bigvee_{x \in a+b} \operatorname{rmin}\left\{\operatorname{rmin}\left\{\widetilde{\mu_{F}}(a), \widetilde{\delta}\right\}, \operatorname{rmin}\left\{\widetilde{\mu_{G}}(b), \widetilde{\delta}\right\}\right\} \\
& \quad \leq \bigvee_{x \in a+b} \operatorname{rmin}\left\{\operatorname{rmax}\left\{\widetilde{\mu_{F}}(a y), \tilde{\gamma}\right\}, \operatorname{rmax}\left\{\widetilde{\mu_{G}}(b y), \tilde{\gamma}\right\}\right\} \\
& \quad=\operatorname{rmax}\left\{\bigvee_{x \in a+b} \operatorname{rmin}\left\{\widetilde{\mu_{F}}(a y), \widetilde{\mu_{G}}(b y)\right\}, \widetilde{\gamma}\right\} \\
& \quad \leq \operatorname{rmax}\left\{\widetilde{\mu_{F \oplus G}}(x y), \tilde{\gamma}\right\} .
\end{aligned}
$$

In a similar way, we have $\operatorname{rmax}\left\{\widetilde{\mu_{F \oplus G}}(x y), \tilde{\gamma}\right\} \geq \operatorname{rmin}$ $\left\{\widetilde{\mu_{F \oplus G}}(y), \widetilde{\delta}\right\}$. Hence, $\operatorname{rmax}\left\{\widetilde{\mu_{F \oplus G}}(x y), \widetilde{\gamma}\right\} \geq \operatorname{rmin}\left\{\widetilde{\mu_{F \oplus G}}(x)\right.$, $\left.\widetilde{\mu_{F \oplus G}}(y), \widetilde{\delta}\right\}$.

Theorem 30. Let $\mathbb{Q} \mathbb{\mathbb { E }}(R)$ be the set of all interval valued $\left(\epsilon_{\tilde{\gamma}}, \epsilon_{\tilde{\gamma}} \vee q_{\tilde{\delta}}\right)$-fuzzy hyperideals of $R$ with the same tip " $t$ " (i.e., $\overline{\mu_{F}}(0)=\widetilde{\mu_{G}}(0)$ for all $\left.F, G \in \mathbb{R} \mathbb{\mathbb { Q }}(R)\right)$. Then $(\mathbb{Q} \mathbb{E}(R), \cap, \oplus, \subseteq$ $\left.\vee q_{(\tilde{\gamma}, \widetilde{\delta})}\right)$ is a modular lattice.

Proof. Let $F, G \in \mathbb{Q} \mathbb{\mathbb { Q }}(R)$. Clearly, $F \cap G$ is the greatest lower bound of $F$ and $G$. By Theorem 29, we have $F \oplus G \in \mathbb{E} \mathbb{E}(R)$. Now, we prove that $F \oplus G=F \vee G$. Let $x \in R$. Since $x=0+x$ 
and $\operatorname{rmax}\left\{\widetilde{\mu_{F}}(0), \widetilde{\gamma}\right\}=\operatorname{rmax}\left\{\widetilde{\mu_{G}}(0), \widetilde{\gamma}\right\} \geq \operatorname{rmin}\left\{\widetilde{\mu_{G}}(y), \widetilde{\delta}\right\}$ for all $y \in R$, we have

$$
\begin{aligned}
& \operatorname{rmax}\left\{\widetilde{\mu_{F \oplus G}}(x), \widetilde{\gamma}\right\} \\
& \quad=\operatorname{rmax}\left\{\bigvee_{x \in y+z} \operatorname{rmin}\left\{\widetilde{\mu_{F}}(y), \widetilde{\mu_{G}}(z)\right\}, \widetilde{\gamma}\right\} \\
& \quad \geq \operatorname{rmax}\left\{\operatorname{rmin}\left\{\widetilde{\mu_{F}}(0), \widetilde{\mu_{G}}(x)\right\}, \widetilde{\gamma}\right\} \\
& =\operatorname{rmin}\left\{\operatorname{rmax}\left\{\widetilde{\mu_{F}}(0), \widetilde{\gamma}\right\}, \operatorname{rmax}\left\{\widetilde{\mu_{G}}(x), \widetilde{\gamma}\right\}\right\} \\
& \quad \geq \operatorname{rmin}\left\{\operatorname{rmin}\left\{\widetilde{\mu_{G}}(x), \widetilde{\delta}\right\}, \operatorname{rmin}\left\{\widetilde{\mu_{G}}(x), \widetilde{\delta}\right\}\right\} \\
& \quad=\operatorname{rmin}\left\{\widetilde{\mu_{G}}(x), \widetilde{\delta}\right\} ;
\end{aligned}
$$

it follows that $F \subseteq \vee q_{(\widetilde{\gamma}, \widetilde{\delta})} F \oplus G$. Similarly, $G \subseteq \vee q_{(\widetilde{\gamma}, \widetilde{\delta})} F \oplus G$ and so $F \oplus G$ is an upper bound of $F$ and $G$. Now, let $H \in$ $\mathbb{Q} \mathbb{E}(R)$ be such that $F \subseteq \vee q_{(\tilde{\gamma}, \widetilde{\delta})} H$ and $G \subseteq \vee q_{(\tilde{\gamma}, \widetilde{\delta})} H$. It is easy to see $F \oplus G \subseteq \vee q_{(\tilde{\gamma}, \widetilde{\delta})} H$. So, $F \oplus G=F \vee G$. Therefore, $\left(\mathbb{Q} \mathbb{\mathbb { Z }}(R), \cap, \oplus, \subseteq \vee q_{(\tilde{\gamma}, \widetilde{\delta})}\right)$ is a lattice.

For modularity, we first let $F, G, H \in \mathbb{R} \mathbb{\mathbb { V }}(R)$ be such that $F \subseteq \vee q_{(\tilde{\gamma}, \widetilde{\delta})} H$. We need to show that $F \cap(G \oplus H) \approx(F \cap G) \oplus H$. But, the relation $(F \cap G) \oplus H \subseteq \vee q_{(\tilde{\gamma}, \widetilde{\delta})} F \cap(G \oplus H)$ is clear. It remains to show that $F \cap(G \oplus H) \subseteq \vee q_{(\tilde{\gamma}, \widetilde{\delta})}(F \cap G) \oplus H$. Now let $x \in R$. Then we have

$$
\begin{aligned}
& \operatorname{rmax}\left\{\widetilde{\mu_{(F \cap G) \oplus H}}(x), \tilde{\gamma}\right\} \\
& =\operatorname{rmax}\left\{\bigvee_{x \in y+z} \operatorname{rmin}\left\{\widetilde{\mu_{F \cap G}}(y), \widetilde{\mu_{H}}(z)\right\}, \widetilde{\gamma}\right\} \\
& =\operatorname{rmax}\left\{\bigvee_{x \in y+z} \operatorname{rmin}\left\{\widetilde{\mu_{F}}(y), \widetilde{\mu_{G}}(y), \widetilde{\mu_{H}}(z)\right\}, \widetilde{\gamma}\right\} \\
& =\bigvee_{x \in y+z} \operatorname{rmin}\left\{\operatorname{rmax}\left\{\widetilde{\mu_{F}}(y), \tilde{\gamma}\right\}, \operatorname{rmax}\left\{\widetilde{\mu_{G}}(y), \tilde{\gamma}\right\},\right. \\
& \left.\operatorname{rmax}\left\{\widetilde{\mu_{H}}(z), \widetilde{\gamma}\right\}\right\} \\
& \geq \bigvee_{x \in y+z} \operatorname{rmin}\left\{\operatorname{rmin}\left\{\widetilde{\mu_{F}}(x), \widetilde{\mu_{F}}(-z), \widetilde{\delta}\right\},\right. \\
& \left.\operatorname{rmax}\left\{\widetilde{\mu_{G}}(y), \widetilde{\gamma}\right\}, \operatorname{rmax}\left\{\widetilde{\mu_{H}}(z), \widetilde{\gamma}\right\}\right\} \\
& =\bigvee_{x \in y+z} \operatorname{rmin}\left\{\operatorname{rmin}\left\{\widetilde{\mu_{F}}(x), \widetilde{\delta}\right\}, \operatorname{rmin}\left\{\widetilde{\mu_{F}}(-z), \widetilde{\delta}\right\}\right. \text {, } \\
& \left.\operatorname{rmax}\left\{\widetilde{\mu_{G}}(y), \widetilde{\gamma}\right\}, \operatorname{rmax}\left\{\widetilde{\mu_{H}}(z), \widetilde{\gamma}\right\}\right\} \\
& =\bigvee_{x \in y+z} \operatorname{rmin}\left\{\operatorname{rmin}\left\{\widetilde{\mu_{F}}(x), \widetilde{\delta}\right\}, \operatorname{rmax}\left\{\widetilde{\mu_{G}}(y), \widetilde{\gamma}\right\}\right. \text {, } \\
& \left.\operatorname{rmax}\left\{\widetilde{\mu_{H}}(z), \widetilde{\gamma}\right\}\right\}
\end{aligned}
$$

(it follows from $F \subseteq \vee q_{(\widetilde{\gamma}, \widetilde{\delta})} H$ that

$$
\left.\operatorname{rmin}\left\{\widetilde{\mu_{F}}(-z), \widetilde{\delta}\right\} \leq \operatorname{rmax}\left\{\widetilde{\mu_{H}}(z), \widetilde{\gamma}\right\}\right)
$$

$$
\begin{aligned}
& \geq \bigvee_{x \in y+z} \operatorname{rmin}\left\{\operatorname{rmin}\left\{\widetilde{\mu_{F}}(x), \widetilde{\delta}\right\}, \operatorname{rmin}\left\{\widetilde{\mu_{G}}(y), \widetilde{\delta}\right\},\right. \\
& \left.\qquad \operatorname{rmin}\left\{\widetilde{\mu_{H}}(z), \widetilde{\delta}\right\}\right\} \\
& =\operatorname{rmin}\left\{\operatorname{rmin}\left\{\widetilde{\mu_{F}}(x), \bigvee_{x \in y+z} \operatorname{rmin}\left\{\widetilde{\mu_{G}}(y), \widetilde{\mu_{H}}(z)\right\}\right\}, \widetilde{\delta}\right\} \\
& \left.\left.=\operatorname{rminin}\left\{\widetilde{\mu_{F \cap(G \oplus H)}}(x), \widetilde{\delta}\right\} . \widetilde{\mu_{G} \oplus H}(x)\right\}, \widetilde{\delta}\right\}
\end{aligned}
$$

It follows that $F \cap(G \oplus H) \subseteq \vee q_{(\widetilde{\gamma}, \widetilde{\delta})}(F \cap G) \oplus H$. Thus $F \cap(G \oplus H) \approx(F \cap G) \oplus H$ and so $\left(\mathbb{V} \mathbb{E}(R), \cap, \oplus, \subseteq \vee q_{(\tilde{r}, \tilde{\delta})}\right)$ is a modular lattice.

\section{Conclusions}

In this paper, we introduced and studied the generalized interval valued $(\alpha, \beta)$-fuzzy hyperideals of a hyperring, with $\alpha, \beta \in\{\epsilon, q, \epsilon \wedge q, \epsilon \vee q\}$ and $\alpha \neq \in \wedge q$, in which special attention was concentrated on the interval valued $\left(\epsilon_{\tilde{\gamma}}, \epsilon_{\tilde{\gamma}} \vee q_{\tilde{\delta}}\right)$-fuzzy hyperideals. As a consequence, some characterizations theorems of interval valued $\left(\epsilon_{\tilde{\gamma}}, \epsilon_{\tilde{\gamma}} \vee q_{\tilde{\delta}}\right)$ fuzzy hyperideals which generalize many results obtained in [21] were provided. Our future work on this topic will focus on studying some other classes of fuzzy interval valued hyperstructure.

\section{Conflict of Interests}

The authors declare that there is no conflict of interests regarding the publication of this paper.

\section{Acknowledgments}

The authors are thankful to the Editor and two anonymous referees for their valuable comments and suggestions that help to improve this paper. This paper was supported in part by the National Natural Science Foundation of China (71301022) and in part by the TianYuan Special Funds of the National Natural Science Foundation of China (11226264).

\section{References}

[1] F. Marty, "Sur une generalization de la notion de groupe," in Proceedings of the 8th Congress Math Scandianaves, pp. 45-49, Stockholm, Sweden, 1934.

[2] P. Corsini, Prolegomena of Hypergroup Theory, Aviani Editore, Tricesimo, 2nd edition, 1993.

[3] T. Vougiouklis, Hyperstructures and Their Representations, Hadronic Press, Palm Harbor, Fla, USA, 1994.

[4] P. Corsini and V. Leoreanu, Applications of Hyperstructure Theory, vol. 5 of Advances in Mathematics, Kluwer Academic Publishers, Dordrecht, The Netherlands, 2003.

[5] L. A. Zadeh, "Fuzzy sets," Information and Computation, vol. 8, pp. 338-353, 1965. 
[6] P. Corsini and V. Leoreanu, "Join spaces associated with fuzzy sets," Journal of Combinatorics, Information \& System Sciences, vol. 20, no. 1-4, pp. 293-303, 1995.

[7] P. Corsini and V. Leoreanu, "Fuzzy sets and join spaces associated with rough sets," Rendiconti del Circolo Matematico di Palermo, vol. 51, no. 3, pp. 527-536, 2002.

[8] B. Davvaz, "Isomorphism theorems of hyperrings," Indian Journal of Pure and Applied Mathematics, vol. 35, no. 3, pp. 321331, 2004.

[9] B. Davvaz and V. Leoreanu-Fotea, Hyperrings Theory and Applications, International Press Academy, 2007.

[10] B. Davvaz and A. Salasi, "A realization of hyperrings," Communications in Algebra, vol. 34, no. 12, pp. 4389-4400, 2006.

[11] B. Davvaz and T. Vougiouklis, "Commutative rings obtained from hyperrings $\left(H_{v}\right.$-rings) with $\alpha^{*}$-relations," Communications in Algebra, vol. 35, no. 11, pp. 3307-3320, 2007.

[12] B. Davvaz and P. Corsini, "Generalized fuzzy hyperideals of hypernear-rings and many valued implications," Iranian Journal of Fuzzy Systems, vol. 17, no. 3, pp. 241-251, 2006.

[13] O. Kazanc1, B. Davvaz, and S. Yamak, "Fuzzy $n$-ary polygroups related to fuzzy points," Computers \& Mathematics with Applications. An International Journal, vol. 58, no. 7, pp. 1466-1474, 2009.

[14] A. Kehagias and K. Serafimidis, "The L-fuzzy Nakano hypergroup," Information Sciences. An International Journal, vol. 169, no. 3-4, pp. 305-327, 2005.

[15] V. Leoreanu, "Direct limit and inverse limit of join spaces associated with fuzzy sets," Pure Mathematics and Applications, vol. 11, no. 3, pp. 509-516, 2000.

[16] V. Leoreanu Fotea, "Fuzzy hypermodules," Computers \& Mathematics with Applications. An International Journal, vol. 57, no. 3, pp. 466-475, 2009.

[17] V. Leoreanu-Fotea and B. Davvaz, "Fuzzy hyperrings," Fuzzy Sets and Systems. An International Journal in Information Science and Engineering, vol. 160, no. 16, pp. 2366-2378, 2009.

[18] M. K. Sen, R. Ameri, and G. Chowdhury, "Fuzzy hypersemigroups," Soft Computing, vol. 12, no. 9, pp. 891-900, 2008.

[19] Y. Yin, J. Zhan, D. Xu, and J. Wang, “The L-fuzzy hypermodules," Computers \& Mathematics with Applications. An International Journal, vol. 59, no. 2, pp. 953-963, 2010.

[20] Y. Yin, B. Davvaz, and J. Zhan, "A fuzzy view of $\Gamma$-hyperrings," Neural Computing and Applications, vol. 21, no. 5, pp. 979-992, 2012.

[21] J. Zhan, B. Davvaz, and K. P. Shum, "Generalized fuzzy hyperideals of hyperrings," Computers \& Mathematics with Applications. An International Journal, vol. 56, no. 7, pp. 17321740, 2008.

[22] J. Zhan, B. Davvaz, and K. P. Shum, "A new view of fuzzy hypernear-rings," Information Sciences. An International Journal, vol. 178, no. 2, pp. 425-438, 2008.

[23] J. Zhan, B. Davvaz, and K. P. Shum, "A new view of fuzzy hyperquasigroups," Journal of Intelligent \& Fuzzy Systems, vol. 20, pp. 147-157, 2009.

[24] J. Zhan, B. Davvaz, and K. P. Shum, "On probabilistic $n$-ary hypergroups," Information Sciences. An International Journal, vol. 180, no. 7, pp. 1159-1166, 2010.

[25] P. M. Pu and Y. M. Liu, "Fuzzy topology. I. Neighborhood structure of a fuzzy point and Moore-Smith convergence," Journal of Mathematical Analysis and Applications, vol. 76, no. 2, pp. 571-599, 1980.
[26] S. K. Bhakat and P. Das, "On the definition of a fuzzy subgroup," Fuzzy Sets and Systems. An International Journal in Information Science and Engineering, vol. 51, no. 2, pp. 235-241, 1992.

[27] A. Rosenfeld, "Fuzzy groups," Journal of Mathematical Analysis and Applications, vol. 35, pp. 512-517, 1971.

[28] S. K. Bhakat and P. Das, “( $\epsilon, \in \vee q)$-fuzzy subgroup," Fuzzy Sets and Systems. An International Journal in Information Science and Engineering, vol. 80, no. 3, pp. 359-368, 1996.

[29] S. K. Bhakat, " $(\in \vee q)$-level subset," Fuzzy Sets and Systems. An International Journal in Information Science and Engineering, vol. 103, no. 3, pp. 529-533, 1999.

[30] S. K. Bhakat, “ $(\epsilon, \in \vee q)$-fuzzy normal, quasinormal and maximal subgroups," Fuzzy Sets and Systems. An International Journal in Information Science and Engineering, vol. 112, no. 2, pp. 299-312, 2000.

[31] Y. Yin and J. Zhan, "New types of fuzzy filters of BL-algebras," Computers \& Mathematics with Applications. An International Journal, vol. 60, no. 7, pp. 2115-2125, 2010.

[32] X. Ma, J. Zhan, and Y. B. Jun, "Some kinds of $\left(\epsilon_{\gamma}, \epsilon_{\gamma} \vee q_{\delta}\right)$ fuzzy ideals of BCI-algebras," Computers \& Mathematics with Applications. An International Journal, vol. 61, no. 4, pp. 10051015, 2011.

[33] X. Ma and J. Zhan, "Characterizations of three kinds of hemirings by fuzzy soft $h$-ideals," Journal of Intelligent \& Fuzzy Systems. Applications in Engineering and Technology, vol. 24, no. 3, pp. 535-548, 2013.

[34] Y. Yin and J. Zhan, "Characterization of ordered semigroups in terms of fuzzy soft ideals," Bulletin of the Malaysian Mathematical Sciences Society, vol. 35, no. 4, pp. 997-1015, 2012.

[35] M. Krasner, "A class of hyperrings and hyperfields," International Journal of Mathematics and Mathematical Sciences, vol. 6, no. 2, pp. 307-311, 1983.

[36] J. Mittas, "Hypergroupes canoniques," Mathematica Balkanica, vol. 2, pp. 165-179, 1972.

[37] L. A. Zadeh, "The concept of a linguistic variable and its application to approximate reasoning-I," vol. 8, pp. 199-249, 1975. 


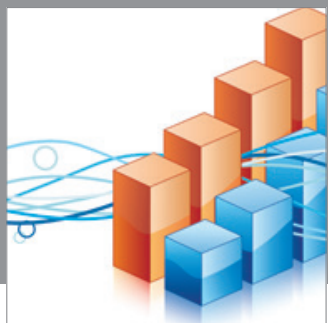

Advances in

Operations Research

mansans

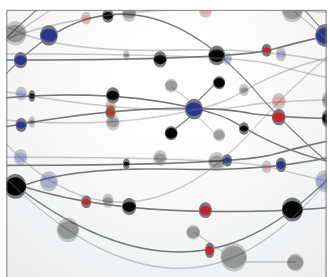

The Scientific World Journal
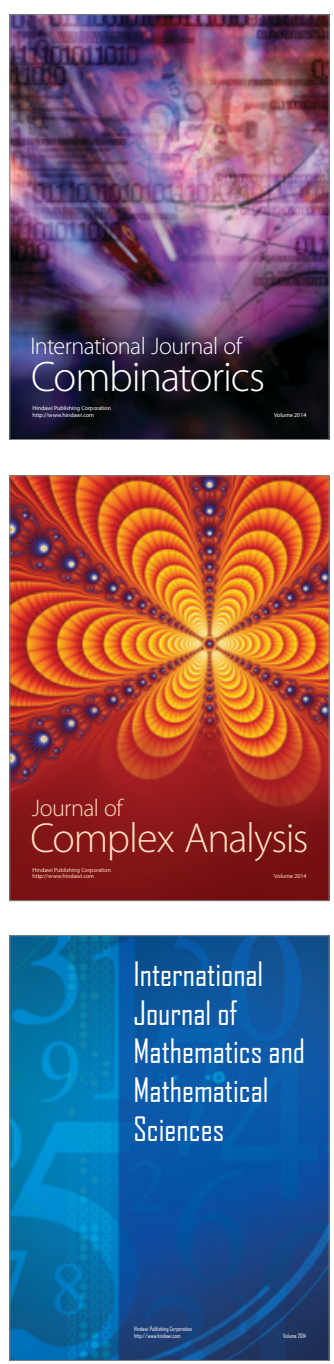
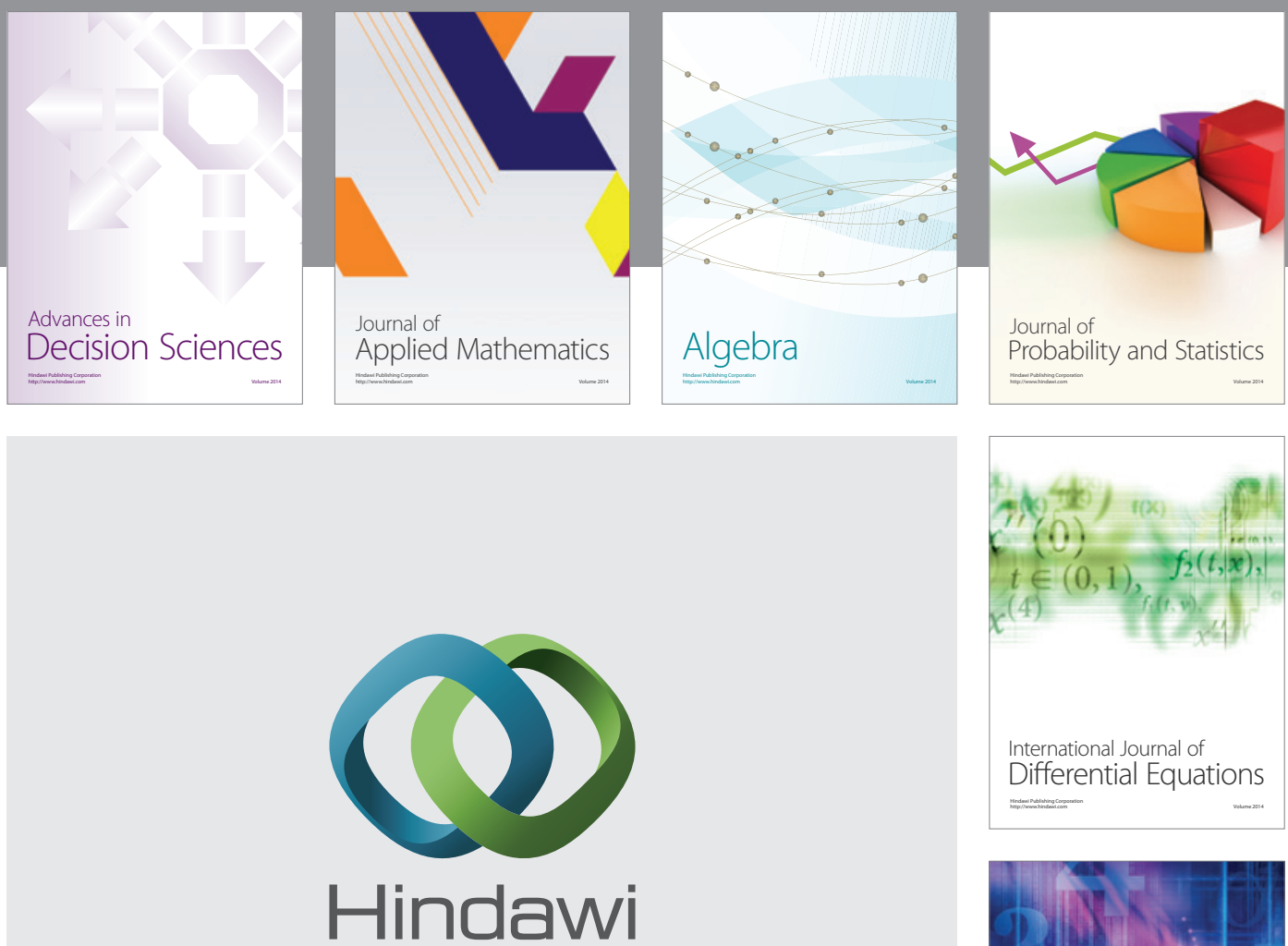

Submit your manuscripts at http://www.hindawi.com
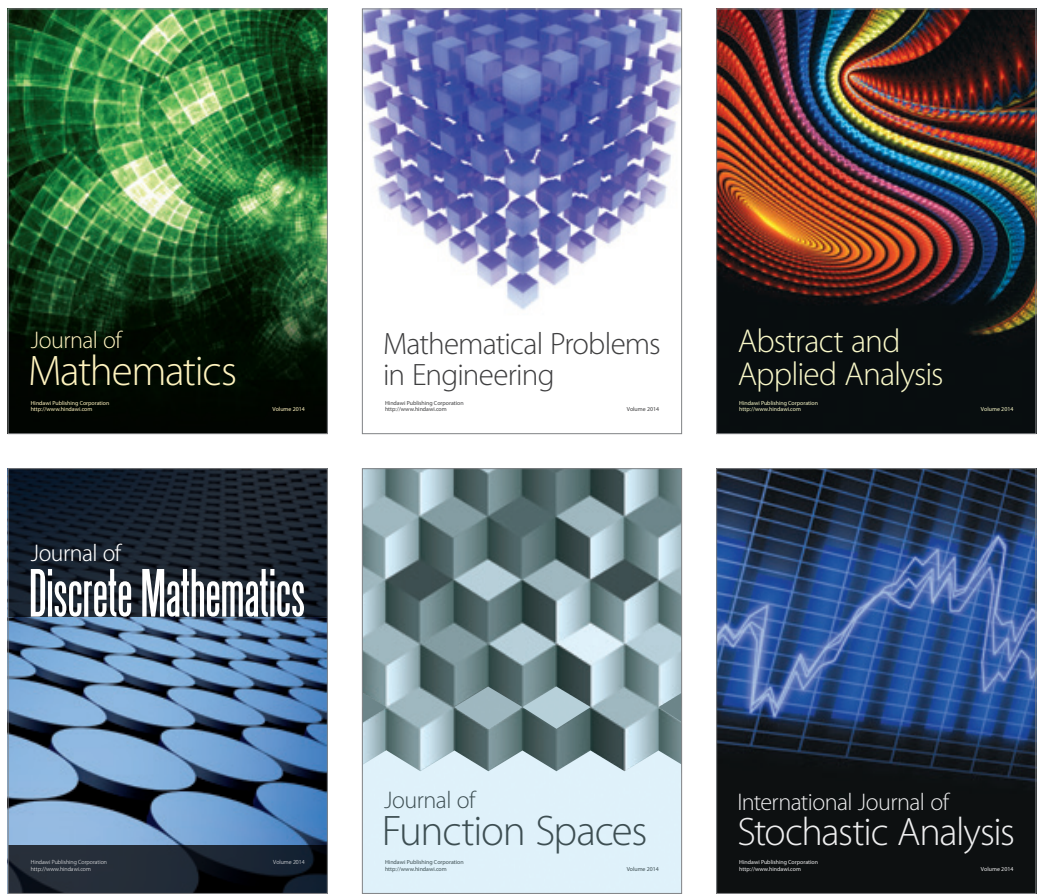

Journal of

Function Spaces

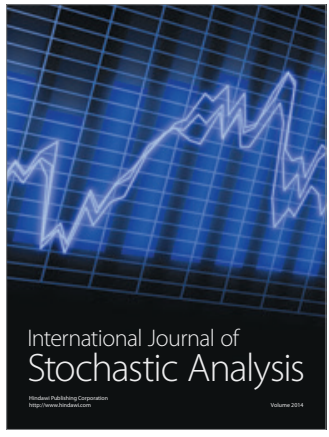

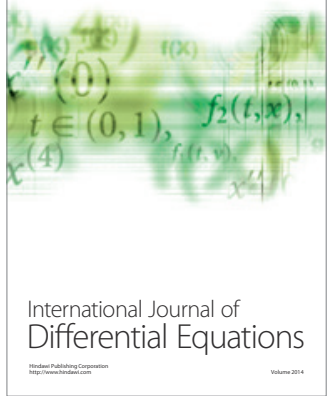
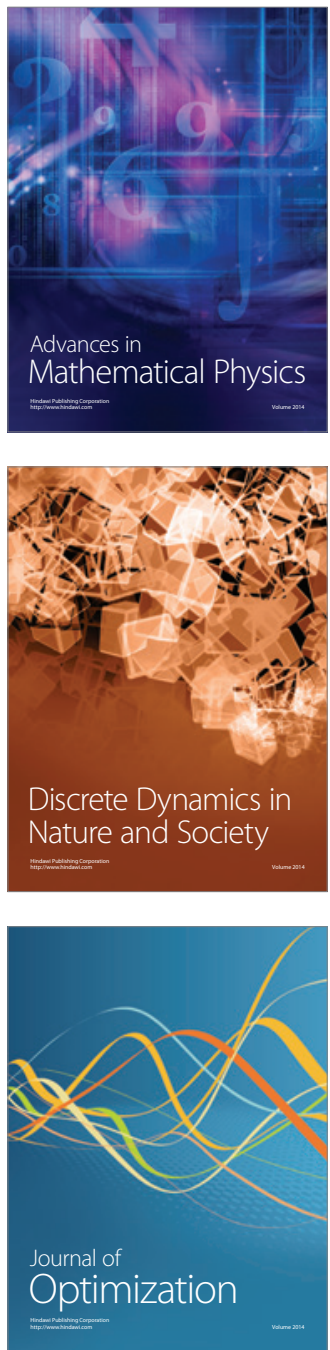